\title{
Damage Location and Characterization Using Matching Pursuit with a Guided Wave Simulation Library
}

\author{
Matthew B. Obenchain* and Carlos E. S. Cesnik ${ }^{\dagger}$ \\ Department of Aerospace Engineering, University of Michigan, Ann Arbor, MI 48109, USA
}

\begin{abstract}
This paper introduces a guided wave signal analysis tool based on chirplet matching pursuit and the local interaction simulation approach (LISA). First, the fundamentals of matching pursuit are described, and the general process for decomposing guided wave time signals is reviewed. Next, a new algorithm is presented that uses a library of LISA simulations to locate and characterize damage in a host plate structure. This algorithm is then demonstrated for a 1-D scenario involving hole damage in both aluminum plates and composite laminates. Experimental sensor results in both material systems are used to reinforce the capability of the method. The remainder of the paper applies the algorithm to locate hole damage in a 2-D scenario, focusing on the cross-ply laminate. Graphical results are presented showing the ability of the new algorithm to predict the location of hole damage using signals from a combination of actuators and sensors.
\end{abstract}

\section{Introduction}

CUIDED wave structural health monitoring (SHM) methods have shown the ability to reliably detect Tdamage in both isotropic and composite structures, and numerous previous studies ${ }^{1}$ have outlined the fundamentals of these techniques. The methods used to analyze guided wave signals and derive useful information from them comprise a significant part of this field of research. These procedures are first intended to identify signal features that result from damage present in the structure. Subsequently, these signal features are used to locate and characterize the damage. Staszewski and Worden ${ }^{2}$ provided a comprehensive review of the numerous dimensions of signal processing used for damage detection.

One of the most prominent categories of processing procedures for guided wave signals is time-frequency analysis. These methods are used to describe how the frequency content of signals change over the time history of the signals, and the information provided can offer insight needed to distinguish and characterize different damage features. Niethammer et al. ${ }^{3}$ provided a good overview of many time-frequency methods such as the short-time Fourier transform and the Wigner-Ville distribution. In addition to these more traditional approaches, wavelet methods have seen increased use in the analysis of guided wave signals. They involve the decomposition of a signal into packets based on some type of basis. Several different bases of wavelets have been used in the literature, but the Morlet (Gabor) and Daubechies wavelets have been the most popular for guided wave decomposition. ${ }^{1} \mathrm{Kim}$ and $\mathrm{Kim}^{4}$ used the continuous wavelet transform to analyze dispersive waves and demonstrated the superiority of the method over the short-time Fourier transform. More recently, Okabe et al. ${ }^{5}$ used the continuous wavelet transform to examine signals from a composite beam containing a delamination. The transform results were used to discern between delaminations of different lengths. Sohn et al. ${ }^{6}$ used the Morlet wavelet to analyze guided wave signals from a damaged composite plate instrumented with an array of sensors.

Mallat and Zhang ${ }^{7}$ introduced the matching pursuit approach, which decomposes a signal into a combination of waveforms from a pre-defined dictionary of time-frequency functions, called atoms. This approach uses an iterative process to select the best matching waveforms. They proposed using a library of Gaussianmodulated time-frequency atoms as the candidate waveforms. Zhang et al. ${ }^{8}$ subsequently demonstrated the

*AIAA Member; Email: mboben@umich.edu

${ }^{\dagger}$ Professor and AIAA Fellow; Email: cesnik@umich.edu; Phone: 1-734-764-3397; Fax: 1-734-763-0578 
use of matching pursuit to de-noise signals. Hong et al. ${ }^{9}$ used matching pursuit analysis in their studies, and they were able to successfully capture very small reflections from a crack in a rod. Das et al. ${ }^{10}$ combined matching pursuits with a Monte Carlo method to reduce the size of the required dictionary and rapidly decompose a signal. Vizzini and Chattopadhyay ${ }^{11}$ used matching pursuit to characterize signals in a delaminated composite panel, and they were able to detect the damage features for a variety of temperatures. Gribonval ${ }^{12}$ introduced an alternative dictionary based on Gaussian-modulated chirplet atoms, which have a linear time-frequency behavior. Raghavan and Cesnik $^{13}$ developed a chirplet matching pursuit approach, and they were able to successfully resolve overlapping, multimodal guided wave signals from damage in aluminum plates. Recently, Agarwal and Mitra ${ }^{14}$ used matching pursuit methods to facilitate machine learning algorithms based on support vector machines and artificial neural networks.

In this work, a damage location and characterization algorithm is introduced that is based on chirplet matching pursuit. Damage difference signals which have been decomposed using matching pursuit are compared to those from a library of simulated damage signals. The damage simulation library is constructed using the local interaction simulation approach. Metrics are developed to match the observed difference signal with the simulation representing the correct location and/or correct damage size. The algorithm is demonstrated for both isotropic and composite plate structures using 1-D and 2-D scenarios.

\section{Theoretical Development}

\section{A. Matching Pursuit Decomposition}

Matching pursuit is a process to decompose signals into a linear combination of waveforms from a pre-defined dictionary. It was originally proposed by Mallat and Zhang, ${ }^{7}$ and it has shown promise in the analysis of guided wave signals. The process of decomposing a signal using matching pursuit is iterative, and a typical iteration $m$ involves first projecting each time-frequency atom $k$ in the dictionary containing $i$ atoms onto the current residual signal $R^{m-1}$. For the first iteration, the residual signal is the original guided wave signal. The best matching atom $k_{i_{m}}$ is chosen as the one which maximizes the value of the projection,

$$
k_{i_{m}}=\underset{k_{i} \in D}{\arg \max }\left|\left\langle R^{m-1}, k_{i}\right\rangle\right|
$$

where the inner product is defined as:

$$
\left\langle f_{1}, f_{2}\right\rangle=\int_{-\infty}^{\infty} f_{1}(t) f_{2}(t) d t
$$

Once the best atom has been identified, the next residual is calculated,

$$
R^{m}=R^{m-1}-\left\langle R^{m-1}, k_{i_{m}}\right\rangle k_{i_{m}}
$$

and the next iteration is begun. With enough iterations, a signal can be completely reconstructed. In practice, it is often the case that only a small number of iterations are needed to capture the important features of a guided wave signal. Noisy signals are easily cleaned by simply stopping the process before enough iterations occur to capture the noise.

\section{B. Choice of Waveform Dictionary}

In their original paper, Mallat and Zhang proposed using a dictionary of Gaussian-modulated time-frequency atoms defined as:

$$
k_{(l, u, \omega)}(t)=\frac{1}{\sqrt{l}} g\left(\frac{t-u}{l}\right) e^{i \omega(t-u)}
$$

where $u$ is the time center of the atom and $\omega$ is the frequency center. The parameter $l$ represents the dilation of the Gaussian window $g$, which is defined as:

$$
g(t)=2^{1 / 4} e^{-\pi t^{2}}
$$

Figure 1 shows the behavior of a typical atom of this type. The time history is shown on the left, and a spectrogram of the signal is shown on the right. From the spectrogram, the stationary time-frequency 


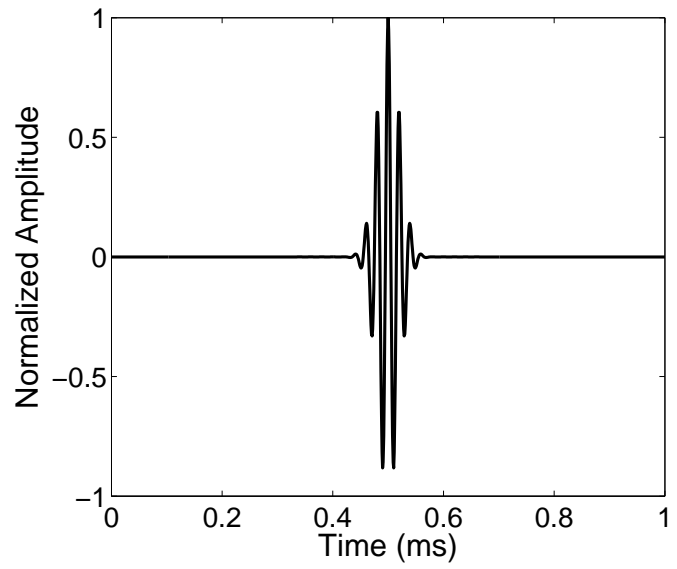

(a) Time History

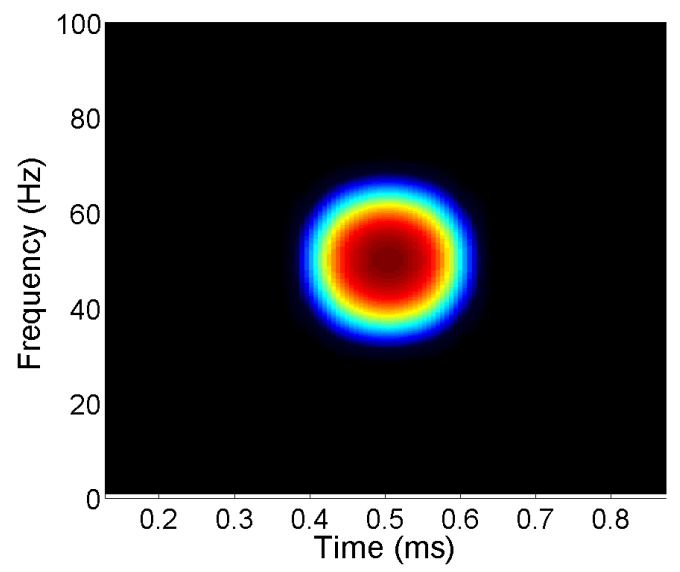

(b) Spectrogram

Figure 1. Stationary Gaussian-modulated atom.

behavior of the atom is apparent, as the frequency at which the peak energy occurs for each time is equal to the center frequency of the atom. Unfortunately, this stationary time-frequency behavior can be problematic for guided wave analysis, since Lamb waves are dispersive in nature.

Gribonval $^{12}$ introduced an alternative waveform dictionary based on Gaussian-modulated chirplets. In this dictionary, an additional parameter $c$ is introduced for the chirp rate of the atom, and the dictionary is defined as:

$$
k_{(l, u, \omega, c)}(t)=\frac{1}{\sqrt{l}} g\left(\frac{t-u}{l}\right) e^{i\left(\omega(t-u)+\frac{c}{2}(t-u)^{2}\right)}
$$

Figure 2 shows the behavior of a chirplet atom. The spectrogram on the right side of this figure shows the non-stationary time-frequency behavior. This chirplet dictionary is better suited for guided wave analysis, since the atoms can account for the frequency dispersion commonly found in guided wave signals. Atoms with higher chirp rates display larger amounts of dispersion, and these atoms produce better matches to guided wave signals containing highly dispersive modes.

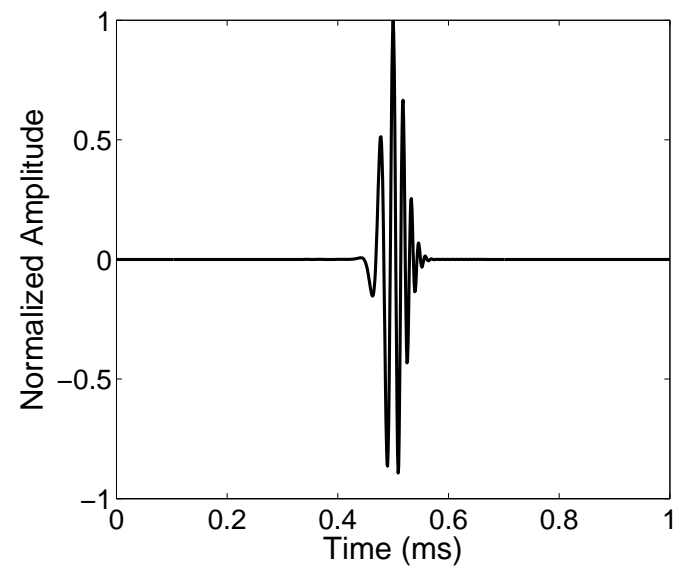

(a) Time History

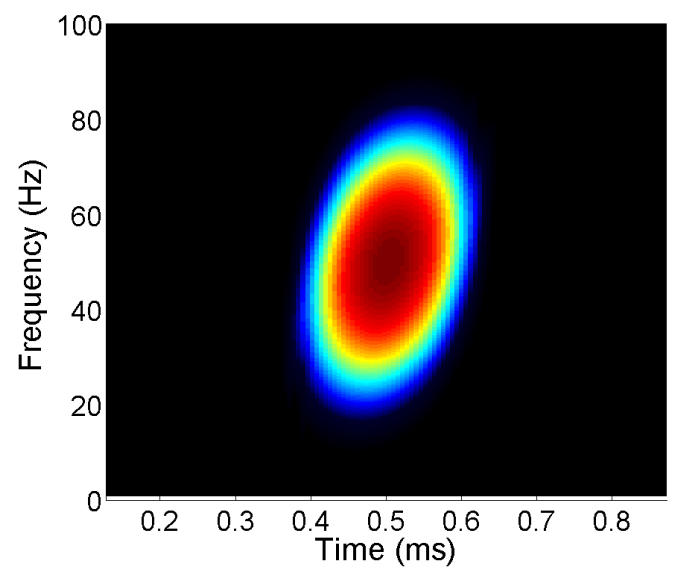

(b) Spectrogram

Figure 2. Gaussian chirplet atom. 


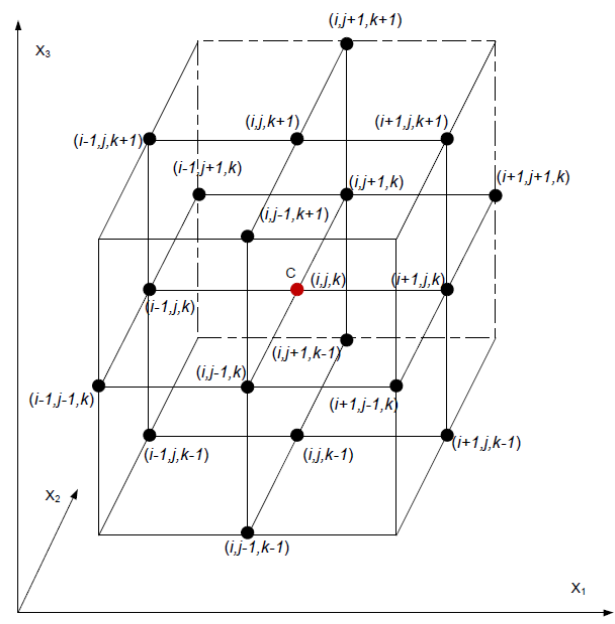

Figure 3. Representation of the 18 neighbors of a generic point in LISA and the Cartesian system used in the analysis. ${ }^{18}$

\section{The Local Interaction Simulation Approach}

The local interaction simulation approach (LISA), originally developed by Delsanto et al., ${ }^{15-17}$ has emerged as an efficient numerical option for modeling guided waves. This method is based on a set of iterative equations for unit cells that discretize the structure, and it uses a sharp interface model to handle material discontinuities and layer interfaces. The displacements of a given point $C$ in a LISA model are only dependent on the displacements of the point's 18 nearest neighbors, shown in Fig. 3, at the previous two time steps. Sinor ${ }^{19}$ presented a thorough derivation of the LISA iterative equations for orthotropic lamina in principal directions which allowed for variable discretization in the three dimensions. Nadella and Cesnik ${ }^{20}$ extended LISA to laminates containing transversely isotropic lamina in non-principal directions and developed a fully-coupled LISA formulation that captures the electromechanical effects of piezoelectric actuators and sensors. ${ }^{21}$ Their work has previously demonstrated LISA's usefulness in modeling a variety of composite configurations including unidirectional, cross-ply, and quasi-isotropic laminates. This work utilizes the LISA Hybrid formulation developed by the authors ${ }^{22}$ to simulate guided wave interaction with damage in the host plate structures. In this formulation, the displacements from a piezo-electric actuator are calculated using the global matrix method, and these displacements are specified in the LISA model at a pre-defined set of points surrounding the actuator region. A schematic of this approach is shown in Fig. 4

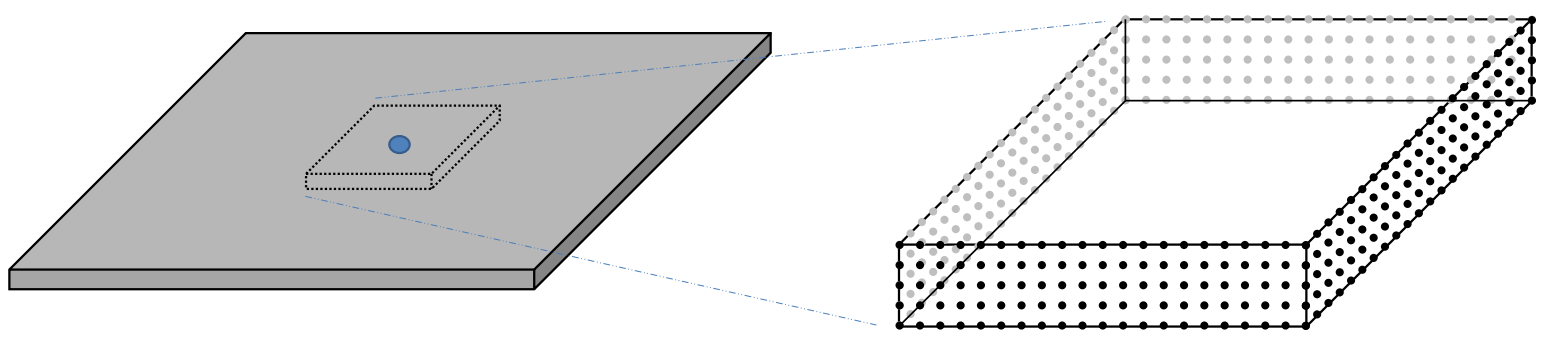

Figure 4. Schematic of displacement input field for LISA hybrid wave propagation model. 


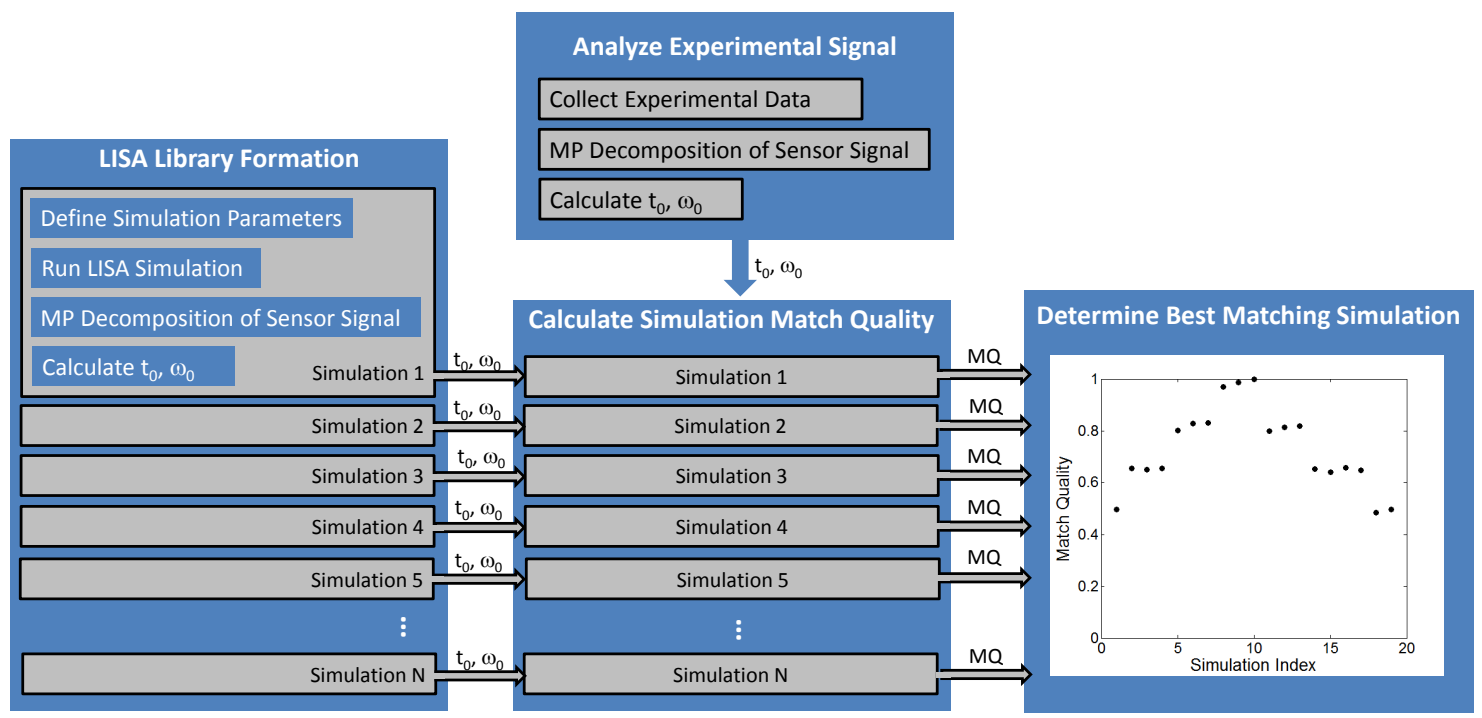

Figure 5. Matching pursuit algorithm flow chart.

\section{Matching Pursuit Algorithm Development}

The literature review in the introduction highlighted several recent implementations of matching pursuit methods. The following algorithm is based on the foundation set by Raghavan and Cesnik, ${ }^{13}$ who introduced the idea of using chirplet matching pursuit along with a library of candidate damage sites to locate damage in simulated or experimental signals. In that work, the scenario assumed a collocated actuator-sensor pair attached to an isotropic structure. Damage was assumed to act as a point scatterer, and an analytical approach was used to develop a library of possible damage signals at known locations, time centers, and frequency centers. The procedure introduced in this paper improves on the previous work by removing the restriction that the actuator and sensor be collocated, and it also allows for analysis of composite laminates with no additional complexity.

A block diagram of the new algorithm is shown in Fig. 5. The first major step in the process is to populate a library of possible damage signals using a series of LISA simulations. This step is a major departure from previous methods, and it eliminates the need to assume that damage sites act as point scatterers. Any damage modes that can be accurately modeled in LISA can be included in the damage library. Each simulation is based on specified parameters including damage location and size. Desired sensor locations can be simulated using the in-plane normal strains contained within the sensor profile, as shown in previous studies. ${ }^{23,24}$ For each simulation, the damage difference signals for each sensor are decomposed using chirplet matching pursuit. The matching pursuit procedure was implemented using the LastWave 3.1 signal processing software, which is available as freeware. ${ }^{25}$ An example result for the matching pursuit decomposition is shown in Fig. 6, where the original sensor signal is shown along with the first atom returned by the decomposition. For this algorithm, the time and frequency centers of the matched atoms, defined as $t_{0}$ and $\omega_{0}$ respectively, are the primary data extracted during the decomposition process.

Once the damage library is complete, a damage difference signal from an experiment or simulation is collected, and it is analyzed using matching pursuit decomposition. The time and frequency centers of the matched atom are recorded. These are passed, along with the time and frequency centers from each simulation in the damage library, to the next step in the process.

The next step is to determine how well each simulated damage case represents the damage captured in the actual difference signal using the parameters obtained in the matching pursuit decomposition. For each simulation, the match error is quantified using:

$$
\text { Match Error }=\frac{\left|t_{0}^{\text {Sim }}-t_{0}^{E x p}\right|}{t_{0}^{E x p}} W+\frac{\left|\omega_{0}^{S i m}-\omega_{0}^{E x p}\right|}{\omega_{0}^{E x p}}(1-W)
$$




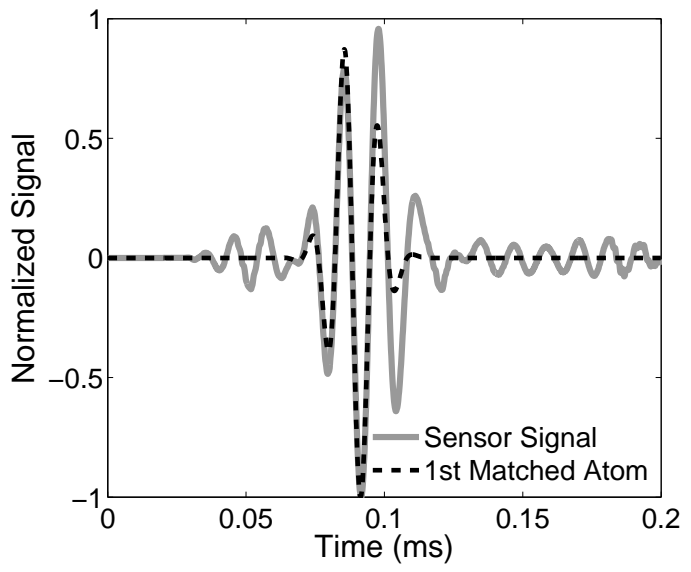

Figure 6. First matched atom for an example damage difference signal.

The $t_{0}^{E x p}$ and $\omega_{0}^{E x p}$ terms represent the time and frequency centers of the matched atom from the actual difference signal, such as one obtained in an experiment. The $t_{0}^{\text {Sim }}$ and $\omega_{0}^{\text {Sim }}$ terms are the time and frequency centers of the matched atom from the library simulation currently under consideration. The parameter $W$ is a weighting factor used to determine how much to consider the difference in frequency centers relative to the difference in time centers. It was determined that using $W=0.25$ produced good results for the cases considered in this study. Once the match error for each damage library simulation is calculated, it is converted to a match quality, $M Q$, metric, i. e.,

$$
M Q(j)=1-\frac{\text { Match Error }(j)}{\text { Max }(\text { Match Error })}
$$

where a match quality value of 1 for simulation $j$ indicates a perfect match between the actual difference signal and the difference signal from simulation $j$. The final step in the algorithm is to compare the match quality of each possible damage simulation to determine which one matches best. The parameters of the best matching simulation can then be reported as the best characterization of the actual damage.

In this study, only the first matched atom from each matching pursuit decomposition was used in the algorithm. This atom corresponded to the unconverted $A_{0}$ portion of the difference signal from each simulated sensor. This algorithm, however, can be extended to take into account the remaining matched atoms, which would capture the unconverted $S_{0}$ mode as well as any mode conversions that are present. Including these additional matched atoms in the match quality calculations should further improve the ability of the algorithm to identify the simulation best matching the experimental damage.

\section{Matching Pursuit Algorithm: 1-D Scenario}

The first analyses using the matching pursuit algorithm focused on locating and characterizing hole damage where the actuator, sensors, and damage were always aligned. A schematic showing the configuration of the actuator and sensors as well as the possible damage locations is shown in Fig. 7. The location of the damage in the library simulations was allowed to vary along a line connecting the actuator, sensor 1, and sensor 2. The hole location varied from $80 \mathrm{~mm}$ from the actuator center to $170 \mathrm{~mm}$ from the actuator center in $5 \mathrm{~mm}$ increments. Additionally, the hole radius was allowed to vary at each location. Holes in both aluminum and cross-ply plates were considered. For the isotropic case, a 700-mm square aluminum host plate with 3.18-mm thickness was modeled. For the cross-ply case, a $[0 / 90]_{3 S}$ laminate constructed with the CYCOM IM7/977-3 material system was used as the host structure. The laminate was a 500$\mathrm{mm}$ square with a thickness of $1.5 \mathrm{~mm}$. Both cases modeled the guided waves generated by a $12.8-\mathrm{mm}$ diameter piezoceramic disc subject to a 3.5 -cycle Hann-modulated toneburst with $75-\mathrm{kHz}$ center frequency. The actuator was assumed to be surface mounted at the geometric center of the plate. The sensors were modeled as piezoceramic discs with a diameter of $7.5 \mathrm{~mm}$. 


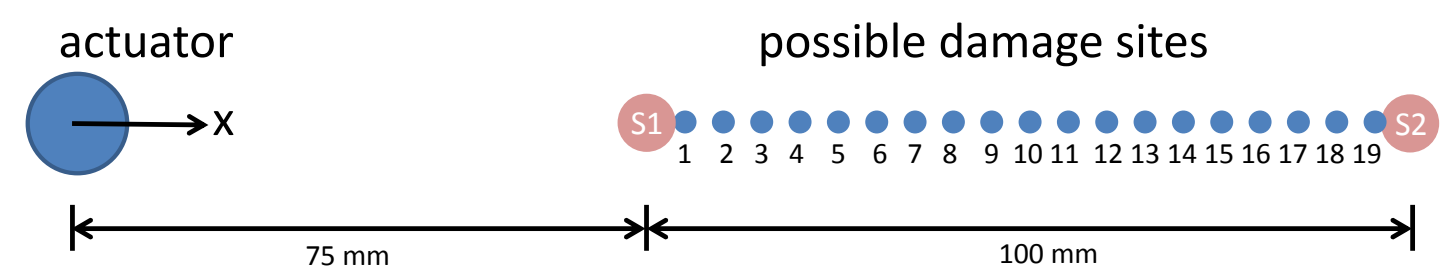

Figure 7. Possible damage locations in matching pursuit damage library (1-D analysis).

\section{A. Identification of Hole Location}

In the first stage of the 1-D analysis, the library of possible damage simulations was restricted to those where the through-thickness hole damage had radius $r=1 t$. The signals representing the actual damage location were taken from one of the simulations, and the algorithm was run to determine if it could identify the correct damage simulation. In these three trial cases, the actual damage locations were at $x=100 \mathrm{~mm}, x$ $=125 \mathrm{~mm}$, and $x=150 \mathrm{~mm}$. Damage difference signals from the two sensors shown in Fig. 7 were used to identify the location of the actual damage. Graphical representations of the matching results for the three simulated damage locations in the aluminum plate are shown in Fig. 8. In each of the figures, the match quality of each damage library simulation is plotted with respect to the simulated damage location. The left column shows the results of the algorithm when data from sensor 1 were used to locate the hole. The right column shows the results when the sensor 2 data were used.

For these three locations, the algorithm correctly identified the simulation corresponding to the actual damage location when information from sensor 1 was used. The algorithm was less successful in clearly identifying the correct damage location using the information from sensor 2. Because the algorithm relies heavily on the value of the time center of the actual and library atoms, the sensor arranged in a pulse-echo configuration relative to the actuator and damage locations performed better than the sensor located in a pitch-catch configuration. In the sensor 2 case, the damage difference signals reached the sensor at nearly the same time regardless of the damage location, so there was not much differentiation between the different library simulations. For sensor 1, the difference signals were primarily reflections from the damage site, and these arrived at the sensor at different times for each damage location.

Similar trials were conducted to determine if the algorithm could locate hole damage in the cross-ply laminate. The actual damage locations were again set to $x=100 \mathrm{~mm}, x=125 \mathrm{~mm}$, and $x=150 \mathrm{~mm}$, and graphical results were produced to show how well each simulation matched the actual damage case. The results are shown in Fig. 9. As in the isotropic case, the matching pursuit algorithm was able to correctly identify the location of the hole damage in each of the three trial locations using the sensor 1 data. The results using the sensor 2 data did not provide a useful location prediction, as was previously seen.

\section{B. Variation of Hole Size}

The next phase of the 1-D analysis restricted the location of the actual damage to $x=125 \mathrm{~mm}$, but the radius of the through-thickness hole was allowed to vary. For the aluminum case, the hole radius varied from $r=0.5 t$ to $r=2 t$ in $0.25 t$ increments for the damage library simulations. The damage difference signal used as the actual result was set to the case where the damage radius was $r=0.5 t, r=1 t$, and $r=1.5 t$ in three different tests of the algorithm. The best results were again obtained using the information from sensor 1, and the results for this sensor are shown in the left column of Fig. 10. In each of the three tests, the algorithm correctly predicted the simulation that matched the actual damage size. Results when data from sensor 2 were used are shown in the right column of Fig. 10. With sensor 2 information, the algorithm correctly identified the size of the $r=0.5 t$ and $r=1 t$ holes. The results were more ambiguous for the $r=1.5 t$ case, where the simulations for $r=1.25 t$ and $r=1.75 t$ were also identified as good matches.

A similar series of steps were taken to determine if the algorithm could identify the damage size for holes in the cross-ply laminate. For the damage library simulations, the hole radius varied from $r=0.5 t$ to $r=3 t$ in $0.5 t$ increments. Three trial cases were run with damage sizes $r=1 t, r=2 t$, and $r=3 t$. The match quality results using sensors 1 and 2 are shown in Fig. 11. For the case where $r=1 t$, data from both sensors 


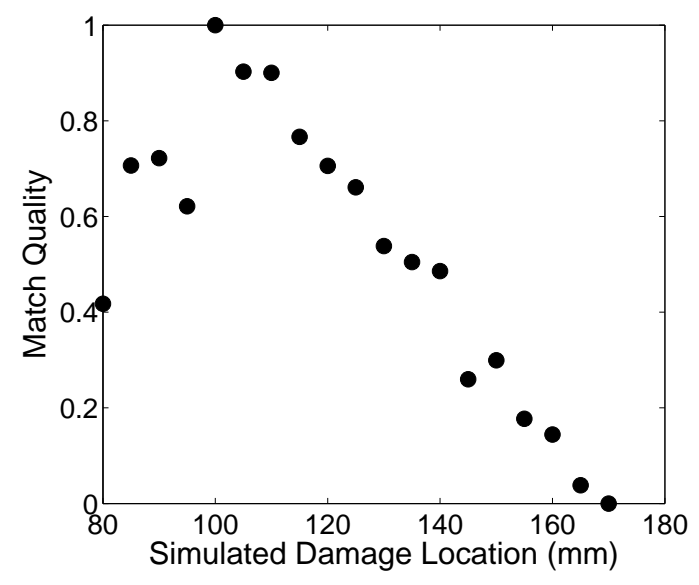

(a) Damage at $x=100 \mathrm{~mm}$ - Sensor 1

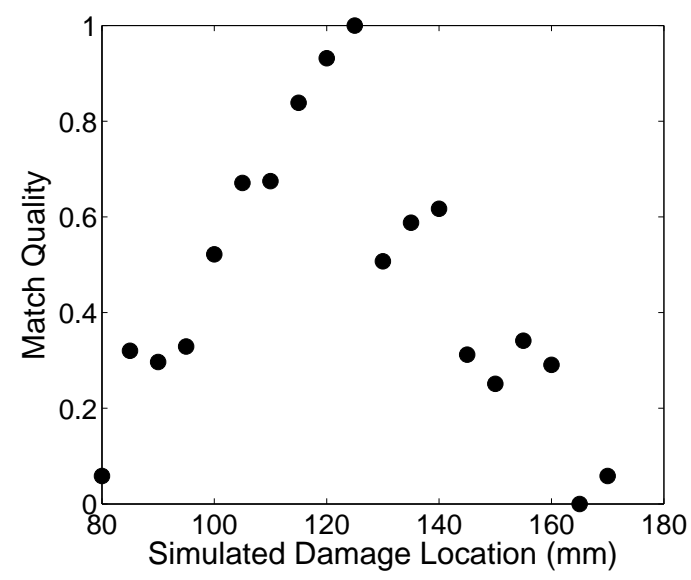

(c) Damage at $x=125 \mathrm{~mm}$ - Sensor 1

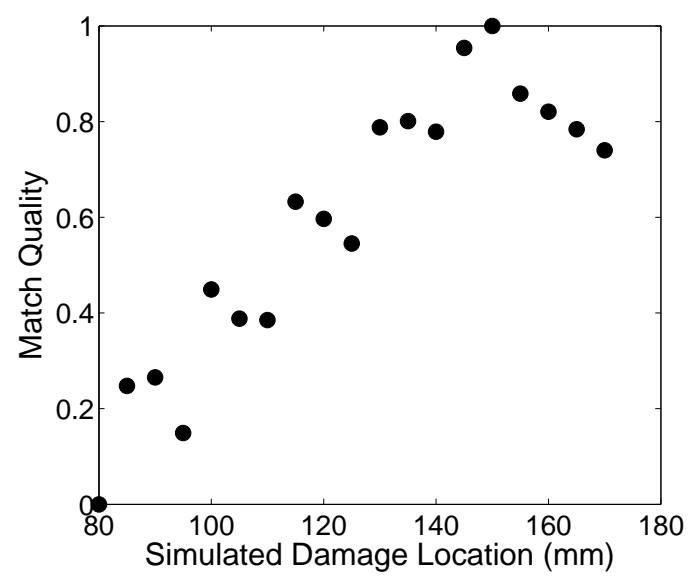

(e) Damage at $x=150 \mathrm{~mm}$ - Sensor 1

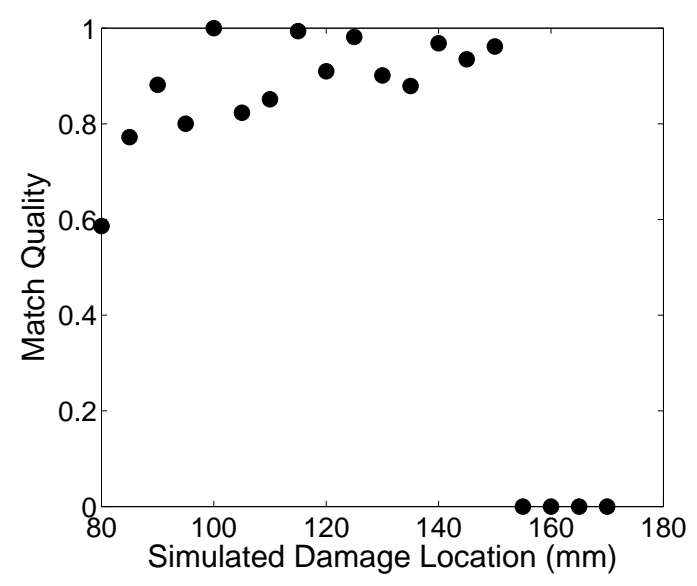

(b) Damage at $x=100 \mathrm{~mm}$ - Sensor 2

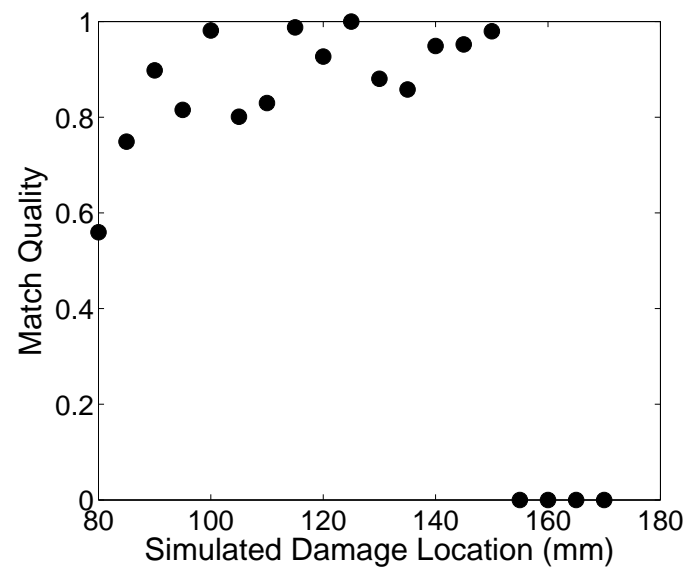

(d) Damage at $x=125 \mathrm{~mm}$ - Sensor 2

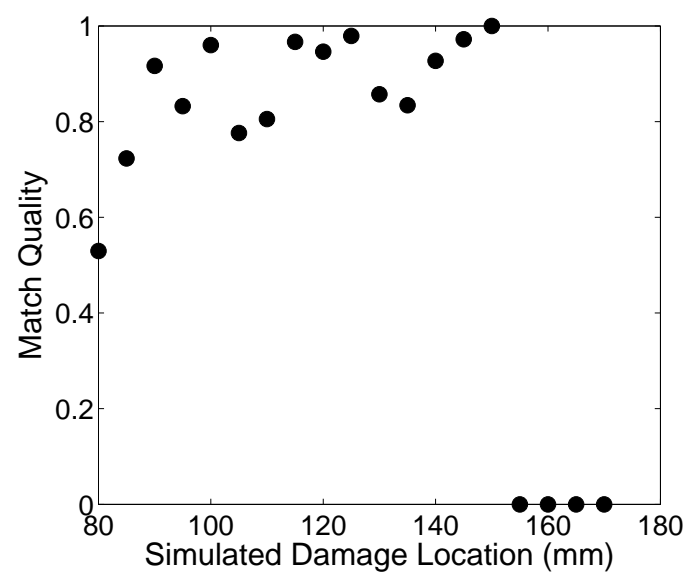

(f) Damage at $x=150 \mathrm{~mm}$ - Sensor 2

Figure 8. Matching pursuit algorithm location results for hole damage in an isotropic plate at various damage sites (1-D analysis). 


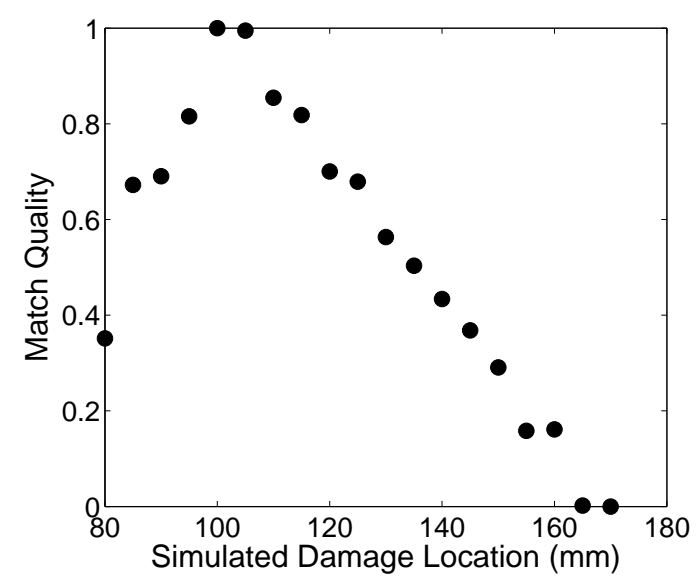

(a) Damage at $x=100 \mathrm{~mm}$ - Sensor 1

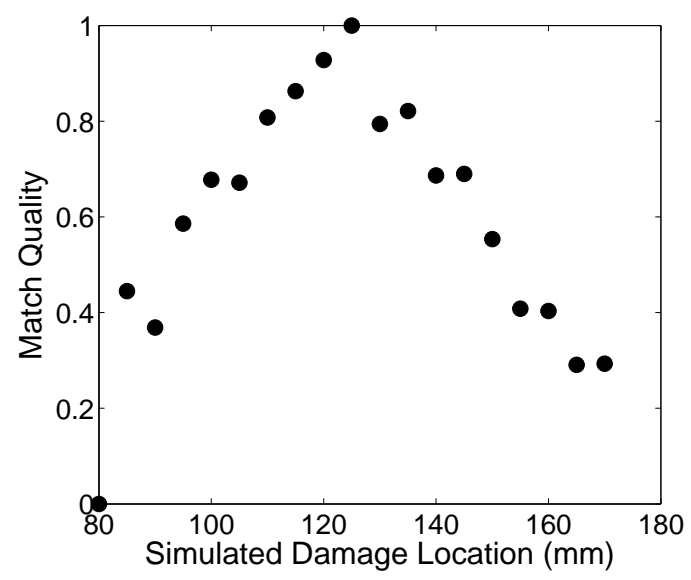

(c) Damage at $x=125 \mathrm{~mm}$ - Sensor 1

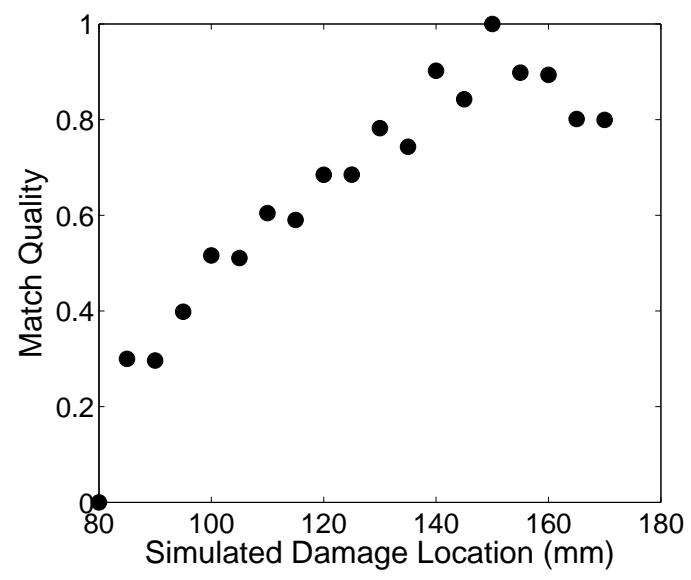

(e) Damage at $x=150 \mathrm{~mm}$ - Sensor 1

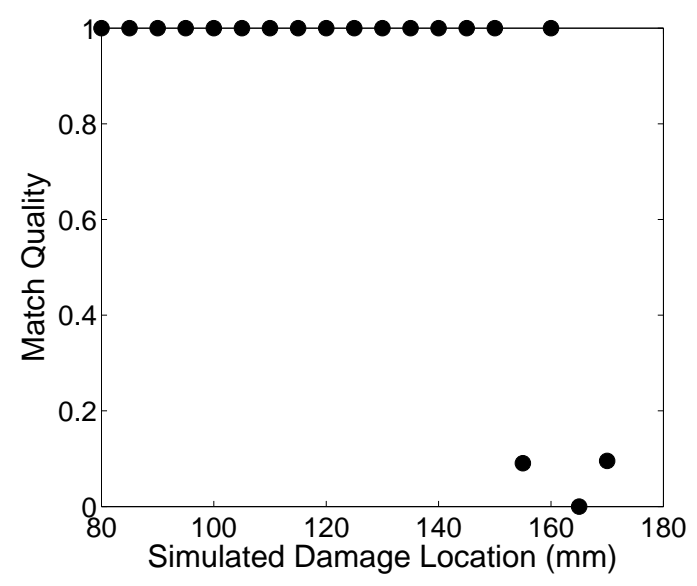

(b) Damage at $x=100 \mathrm{~mm}$ - Sensor 2

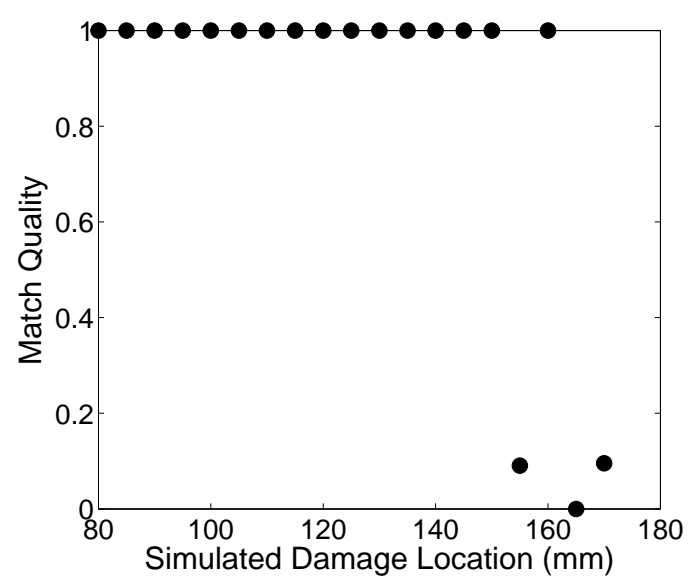

(d) Damage at $x=125 \mathrm{~mm}$ - Sensor 2

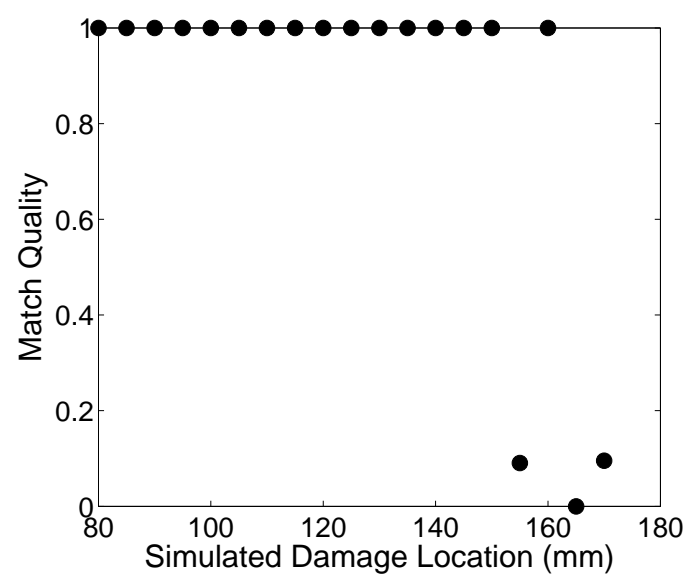

(f) Damage at $x=150 \mathrm{~mm}$ - Sensor 2

Figure 9. Matching pursuit algorithm location results for hole damage in a cross-ply laminate at various damage sites (1-D analysis). 


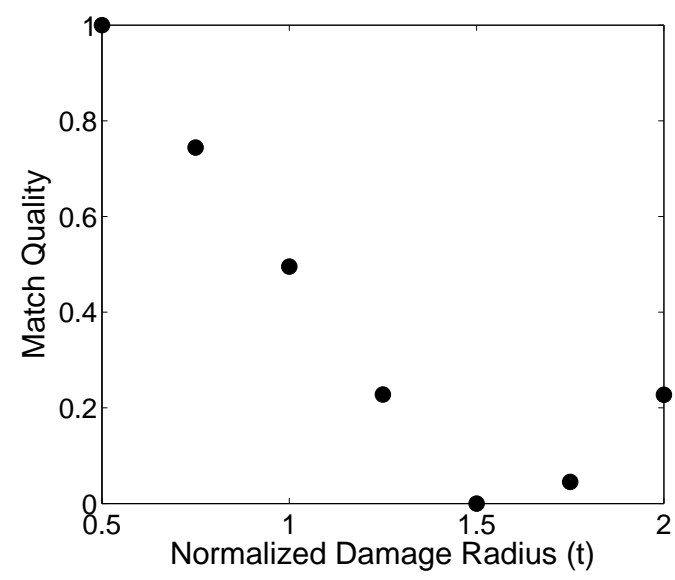

(a) Damage size $r=0.5 t$ - Sensor 1

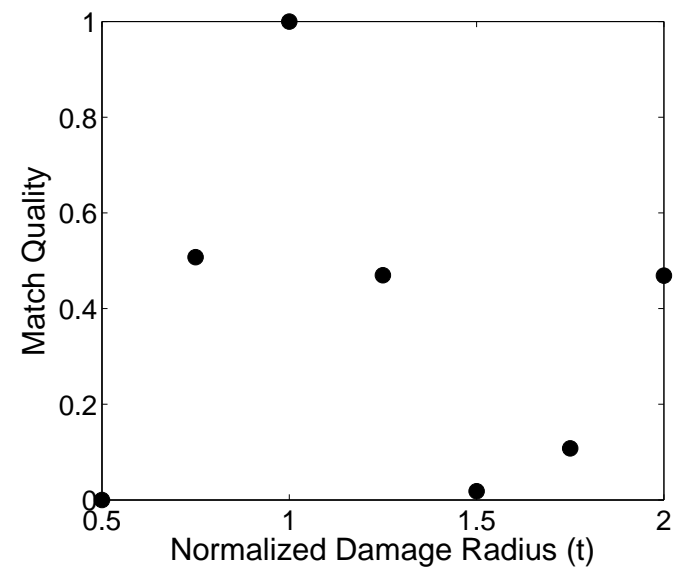

(c) Damage size $r=1 t$ - Sensor 1

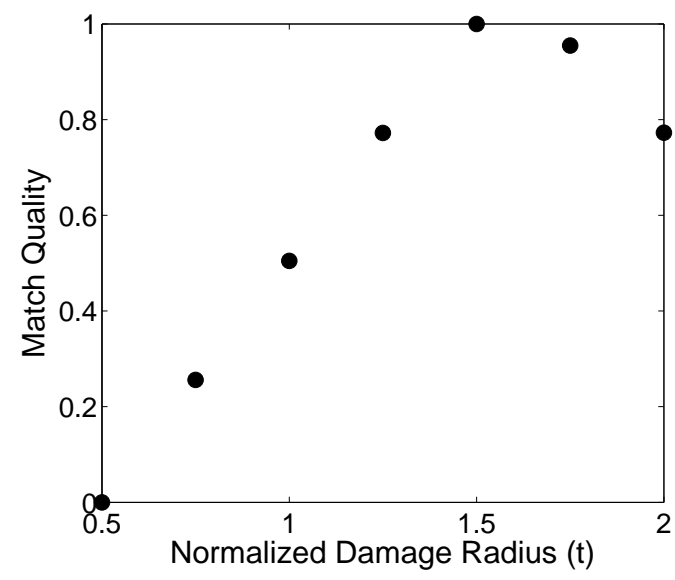

(e) Damage size $r=1.5 t$ - Sensor 1

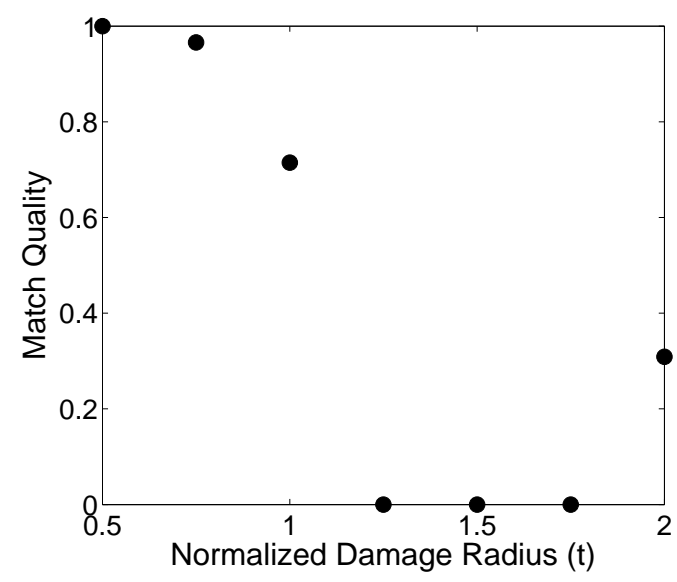

(b) Damage size $r=0.5 t$ - Sensor 2

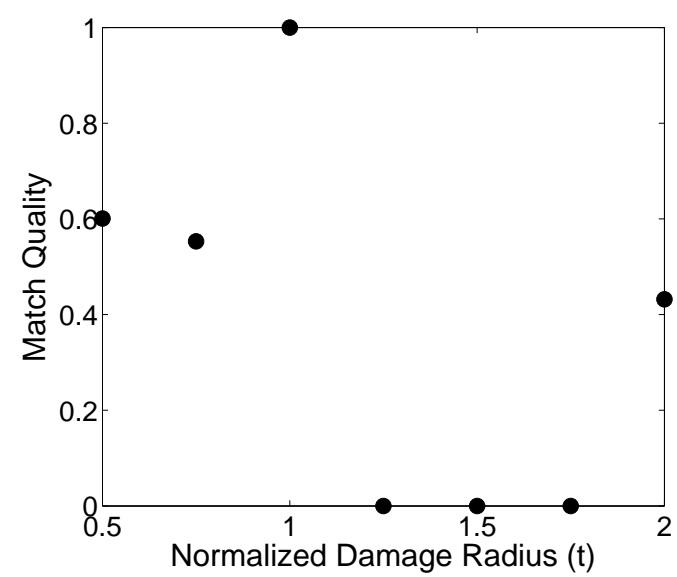

(d) Damage size $r=1 t$ - Sensor 2

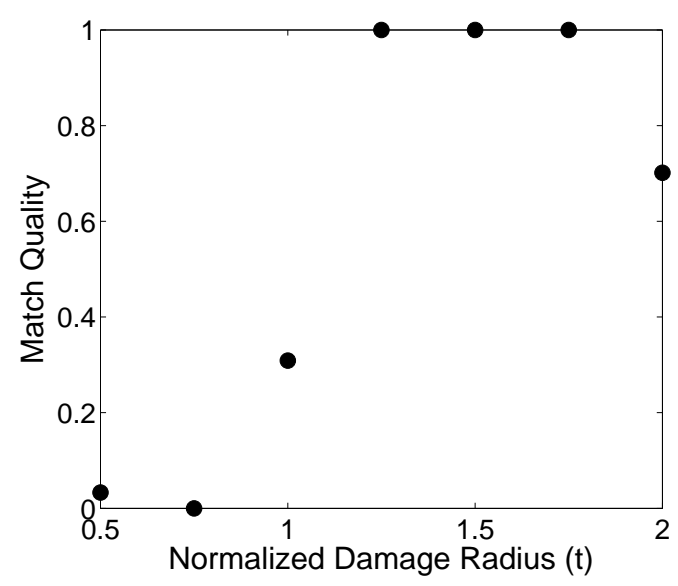

(f) Damage size $r=1.5 t$ - Sensor 2

Figure 10. Matching pursuit algorithm size identification results for hole damage in an isotropic plate with varying radius (1-D analysis). 


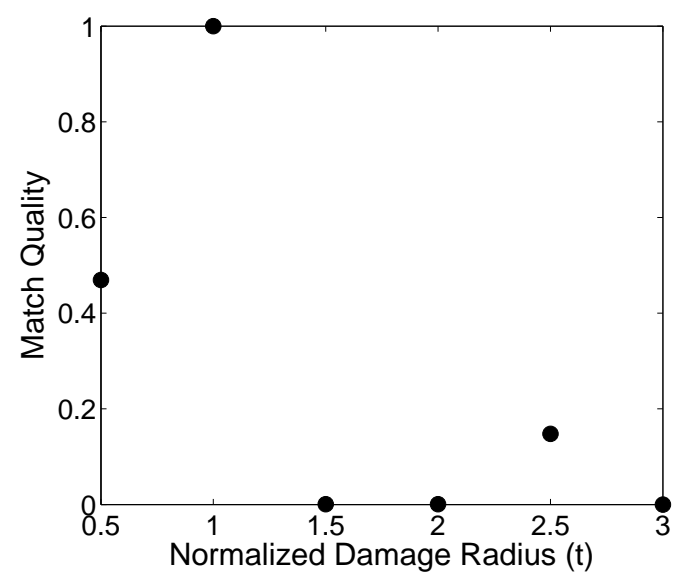

(a) Damage size $r=1 t$ - Sensor 1

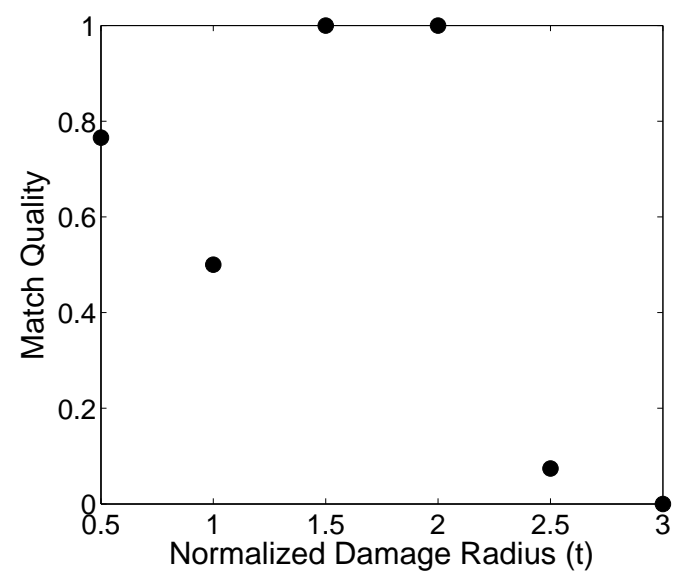

(c) Damage size $r=2 t$ - Sensor 1

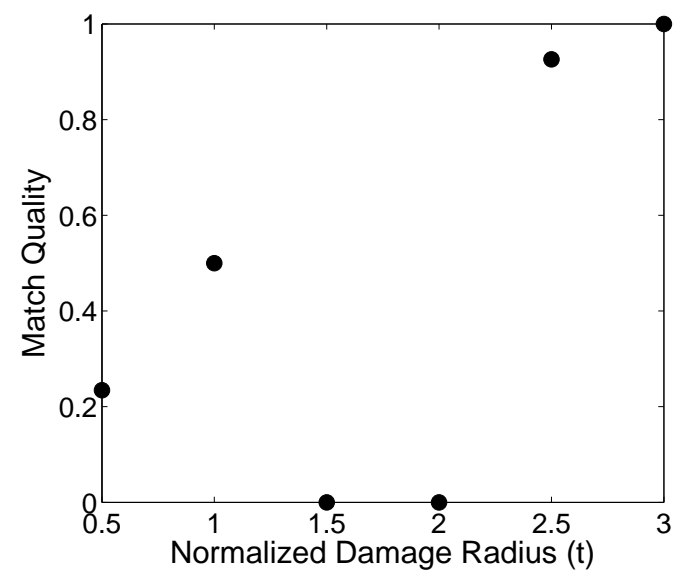

(e) Damage size $r=3 t$ - Sensor 1

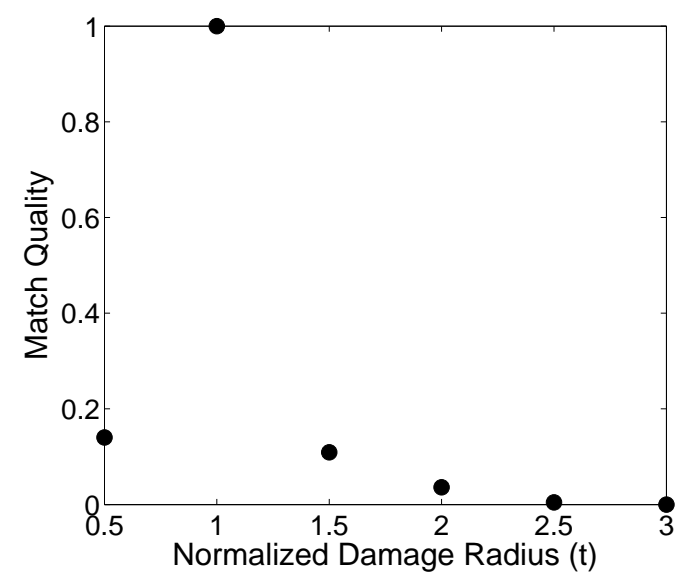

(b) Damage size $r=1 t$ - Sensor 2

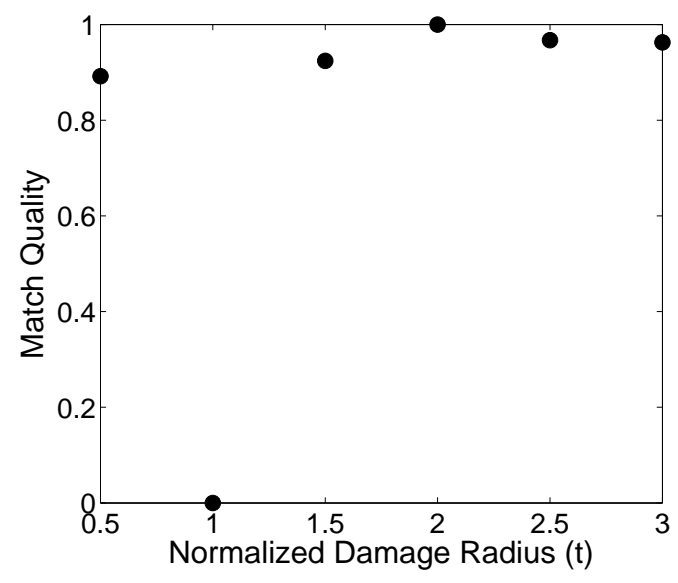

(d) Damage size $r=2 t$ - Sensor 2

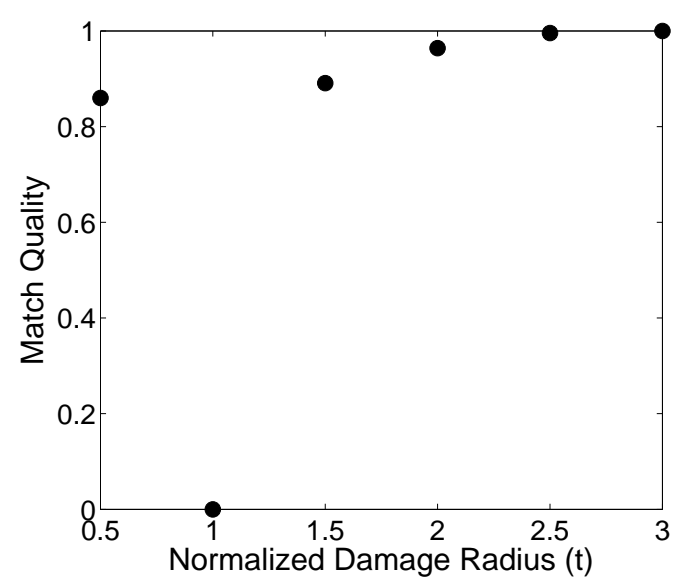

(f) Damage size $r=3 t$ - Sensor 2

Figure 11. Matching pursuit algorithm size identification results for hole damage in a cross-ply laminate with varying radius (1-D analysis). 
produced a correct match for the hole size. This was also true for the case where $r=3 t$. In the case where the hole had radius $r=2 t$, the sensor 2 data produced a correct match, but the sensor 1 data predicted both the $r=1.5 t$ and the $r=2 t$ simulations as perfect matches.

Two observations are notable for the hole variation trials. First, unlike the hole location trials, the sensor 2 data produced correct matches in most cases. The second observation is that the algorithm sometimes produced ambiguous results for the size identification, where hole sizes close to the actual size were also reported as good matches. This result stems from the fact that for a given hole location, the damage difference signals were best represented with atoms with the same time center but slightly different frequency centers. In cases where ambiguous size identification results were produced, the frequency centers of the best matched atoms for two adjacent hole sizes where nearly identical, resulting in similar match quality results.

\section{Variation of Hole Size and Location}

The third phase of the 1-D analysis considered a damage library where three possible hole sizes existed for each possible damage location in the aluminum plate. The hole radii $r=0.5 t, r=1 t$, and $r=2 t$ were included in the library. Various combinations of hole size and location were then assigned as the actual damage case, and the algorithm produced graphical matching results for each case. Only the sensor 1 data were used in this phase of the analysis. Figure 12 shows these results for nine simulated damage signals. In the figures, higher predicted match qualities are represented by darker regions in the figure, with a value of 1.0 indicating a perfect match. The actual parameters of the damage are annotated for each case. To better understand the performance of the algorithm, numerical results were also produced to quantify how well the hole location and hole size were identified. The predicted value for the location of the hole in each case was calculated using a weighted average of the locations corresponding to the two best matching library simulations:

$$
x_{\text {predicted }}=\frac{\sum_{j=1}^{2}\left(x_{\text {sim }}(j)\right)(M Q(j))}{\sum_{j=1}^{2} M Q(j)}
$$

A similar calculation was performed to calculate the predicted radius of the hole. The results for the 9 simulations in Fig. 12 are shown in Table 1. In each case, the damage location was identified within 5 mm of the actual location. The correct hole size was identified in all but one trial.

\section{Robustness to Noise}

The previous three subsections used simulated damage difference signals to represent the true damage, which did not contain noise expected in real-world scenarios. To demonstrate the algorithm's ability to deal with noisy signals, Gaussian white noise with a signal-to-noise ratio of $175 \mathrm{~dB}$ was added to the damage difference

Table 1. Damage characterization results for 1-D matching pursuit algorithm with variable hole size and hole location. All values are measured in $\mathbf{m m}$.

\begin{tabular}{ccccccc}
\hline Trial & $x_{\text {actual }}$ & $x_{\text {predicted }}$ & $x_{\text {error }}$ & $r_{\text {actual }}$ & $r_{\text {predicted }}$ & $x_{\text {actual }}$ \\
\hline 1 & 100 & 105.0 & 5.0 & 0.5 & 0.5 & 0 \\
2 & 100 & 105.0 & 5.0 & 1.0 & 1.5 & 0.5 \\
3 & 100 & 97.5 & 2.5 & 2.0 & 2.0 & 0 \\
4 & 125 & 122.5 & 2.5 & 0.5 & 0.5 & 0 \\
5 & 125 & 122.6 & 2.4 & 1.0 & 1.0 & 0 \\
6 & 125 & 122.5 & 2.5 & 2.0 & 2.0 & 0 \\
7 & 150 & 154.9 & 4.9 & 0.5 & 0.5 & 0 \\
8 & 150 & 147.5 & 2.5 & 1.0 & 1.0 & 0 \\
9 & 150 & 147.6 & 2.4 & 2.0 & 2.0 & 0 \\
\hline
\end{tabular}




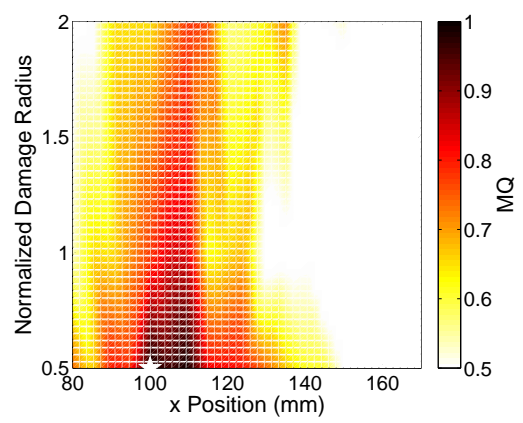

(a) $x=100 \mathrm{~mm}, r=0.5 t$

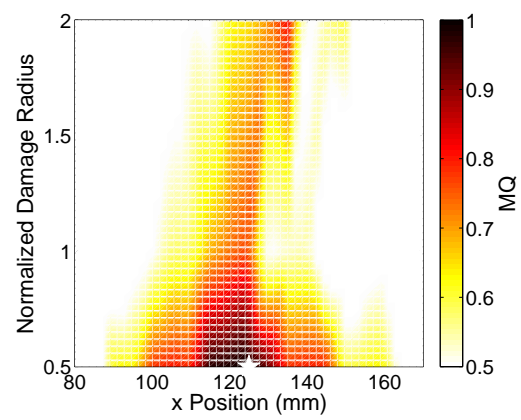

(d) $x=125 \mathrm{~mm}, r=0.5 t$

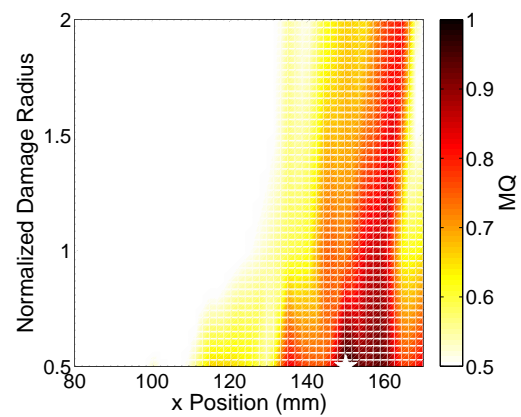

(g) $x=150 \mathrm{~mm}, r=0.5 t$

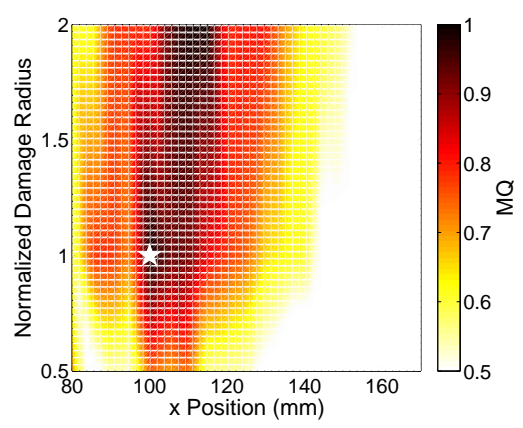

(b) $x=100 \mathrm{~mm}, r=1 t$

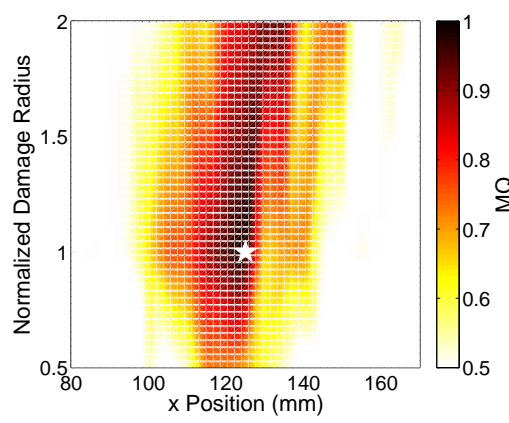

(e) $x=125 \mathrm{~mm}, r=1 t$

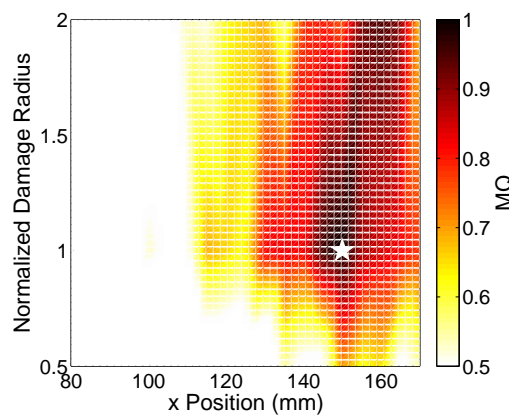

(h) $x=150 \mathrm{~mm}, r=1 t$

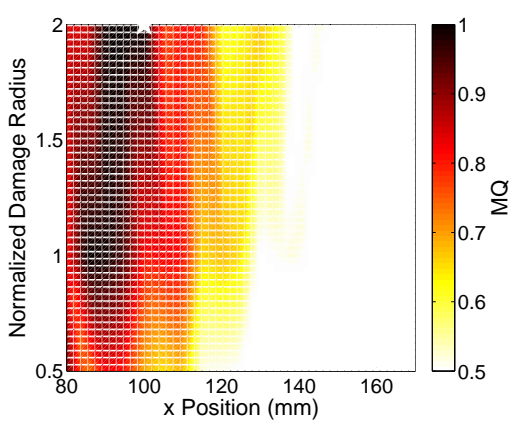

(c) $x=100 \mathrm{~mm}, r=2 t$

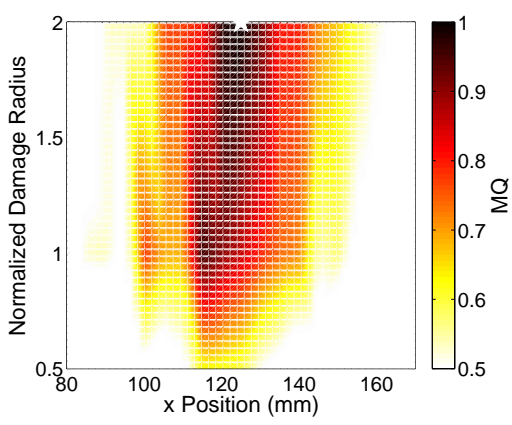

(f) $x=125 \mathrm{~mm}, r=2 t$

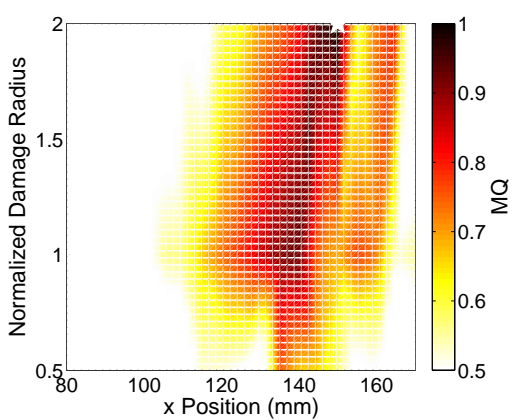

(i) $x=150 \mathrm{~mm}, r=2 t$

Figure 12. Matching pursuit algorithm damage identification results for hole damage in an aluminum plate (1-D analysis, sensor 1 only).

signal from sensor 1 for the case where an $r=1 t$ hole was located at $x=125 \mathrm{~mm}$. In Fig. 13, sub-figures (a) and (b) show the original difference signal and the difference signal with the noise added. When both of these difference signals were used in the matching pursuit algorithm, the resulting matched atoms were almost identical, as shown in sub-figures (c) and (d). While the shape of the atoms was slightly different, their time and frequency centers were almost a perfect match. The location matching results using the original and noisy signals are included in sub-figures (e) and (f). Although the match quality was not as strong, the correct damage location was predicted when the noisy signal was used. Because the matching pursuit algorithm compares the characteristics of the matched atoms and not the damage difference signals themselves, even noisy signals can produce useful information, as long as the noise is uncorrelated. For correlated noise, a significant loss in match quality is likely.

\section{E. Experimental Validation}

The final phase in evaluating the matching pursuit algorithm for the 1-D scenario was to use experimental results for the actual damage difference signals. For this analysis, the experimental data came from two hole 


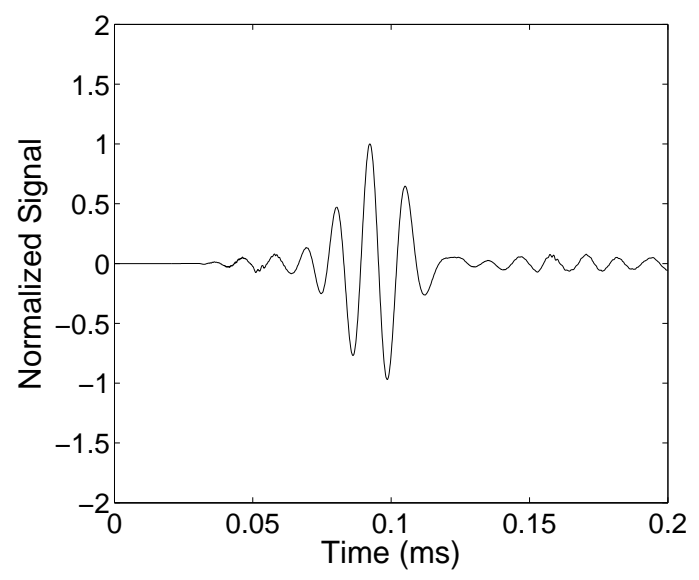

(a) Original sensor 1 difference signal

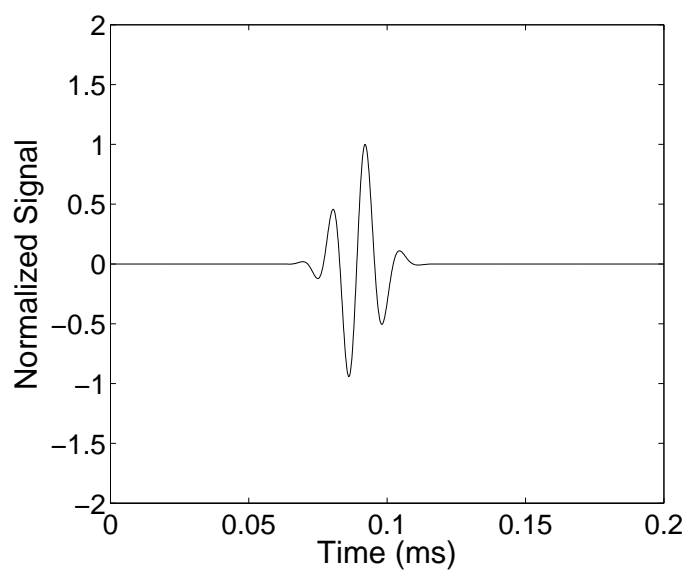

(c) First matched atom for original signal

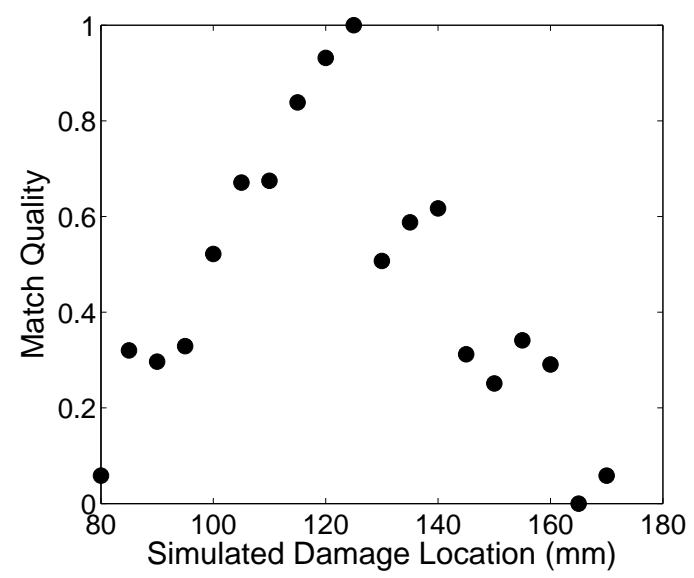

(e) Matching results with original signal

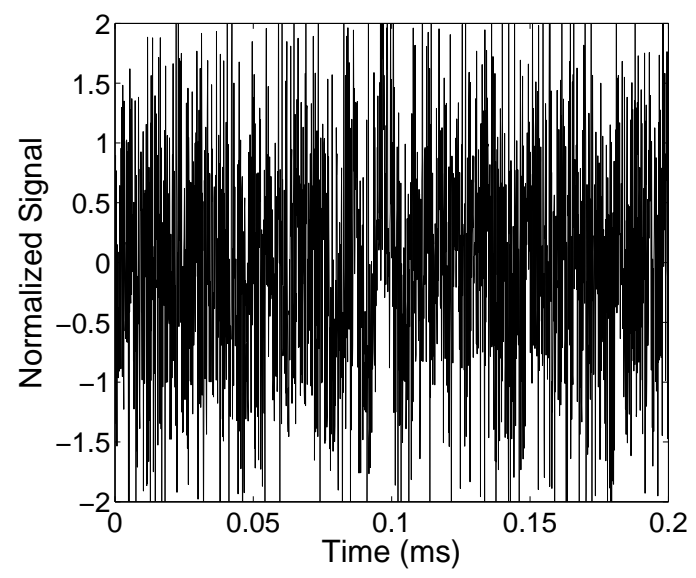

(b) Difference signal with added noise

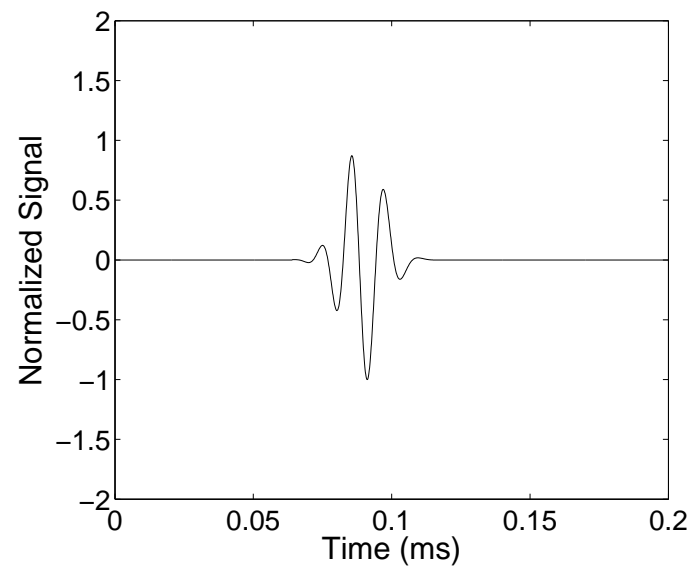

(d) First matched atom for noisy signal

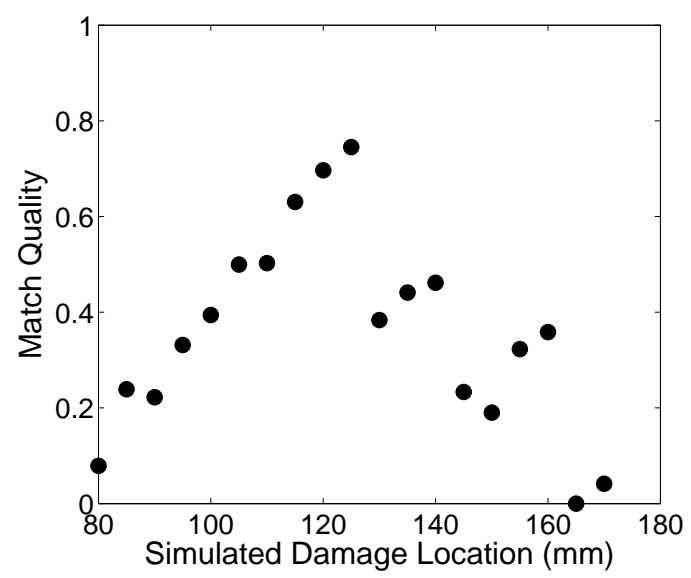

(f) Matching results with noisy signal

Figure 13. Matching pursuit algorithm performance with a noisy sensor signal (isotropic plate with $r=1 t$ hole at $125 \mathrm{~mm}$ from the actuator center). 
experiments with parameters matching those already described in this section. For both the aluminum plate and the cross-ply laminate, a through-thickness hole with radius $r=1 t$ was located at $x=125 \mathrm{~mm}$ relative to the actuator center. The actuator and sensor configurations for both experiments are shown in Fig. 14. For the present analysis only the sensor 1 data were used.

The results for the aluminum plate experiment are shown in Fig. 15. When considering only hole location, the algorithm predicted the hole damage to be best matched with the simulation with damage at $120 \mathrm{~mm}$. When hole size was the only parameter, the correct hole size of $r=1 t$ was successfully predicted. Figure 15 (c) shows a graphical depiction of the results obtained when using the combined library containing three possible sizes for each location. When Eq. 9 was used to determine an overall location prediction based on the two best matching simulations, the hole was predicted to be located at $122.5 \mathrm{~mm}$, which is $2.5 \mathrm{~mm}$ from the actual location.

Results for the cross-ply laminate are shown in Fig. 16. In this case, there was more substantial error between the algorithm's location prediction and the actual damage location. The algorithm identified the simulations with damage at $x=100 \mathrm{~mm}$ and $x=105 \mathrm{~mm}$ as the best matches, which was $20-25 \mathrm{~mm}$ from the actual damage location. An analysis of the sensor signals from the experiment and the simulations showed that the arrival times of the pristine signals correlated well between the experiment and the $x=$ $125 \mathrm{~mm}$ damage simulation. However, the damage difference signal in the experiment arrived sooner than what was predicted in the simulation. It is possible that the process of drilling the hole introduced some internal damage in the laminate that extended closer to the sensor, resulting in an early arrival of the damage difference signal. For the hole size identification analysis, the algorithm was able to successfully predict the size of the hole from the six possible sizes in the damage library.

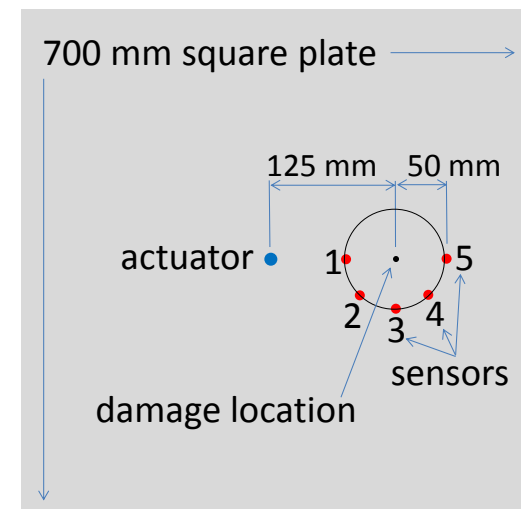

(a) Aluminum experiment configuration

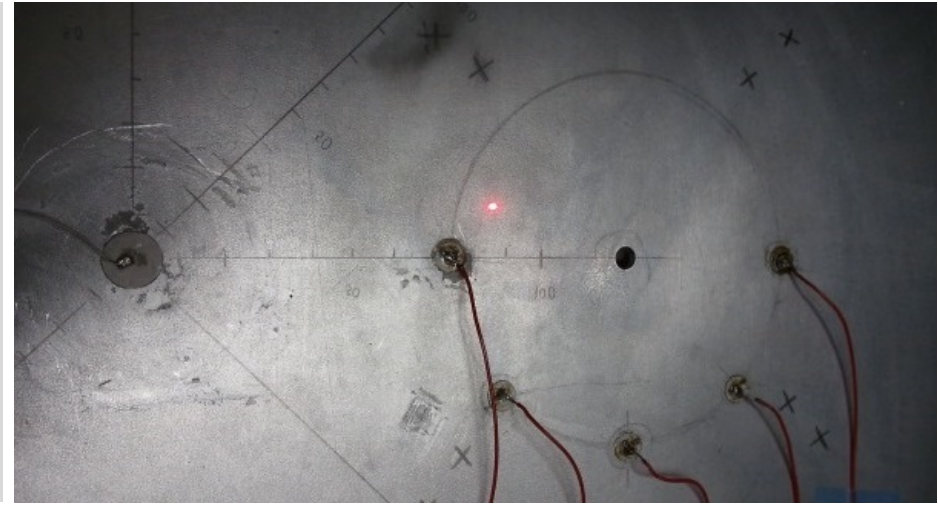

(b) Instrumented aluminum plate

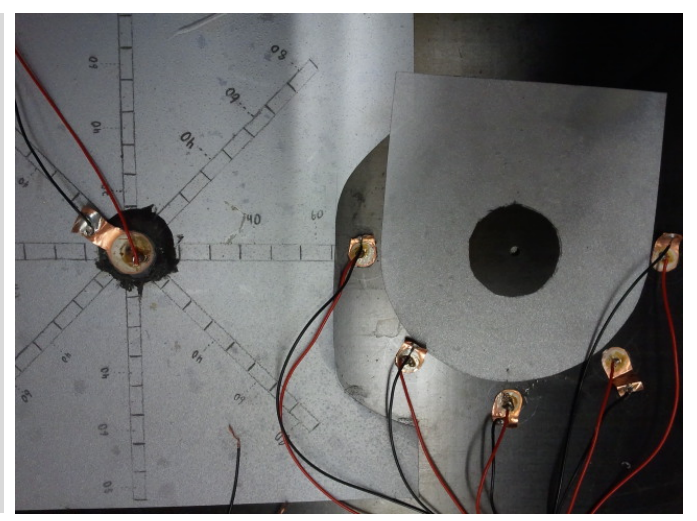

(d) Instrumented crossply plate

(c) Crossply experiment configuration

Figure 14. Experimental configurations of hole damage experiments. 


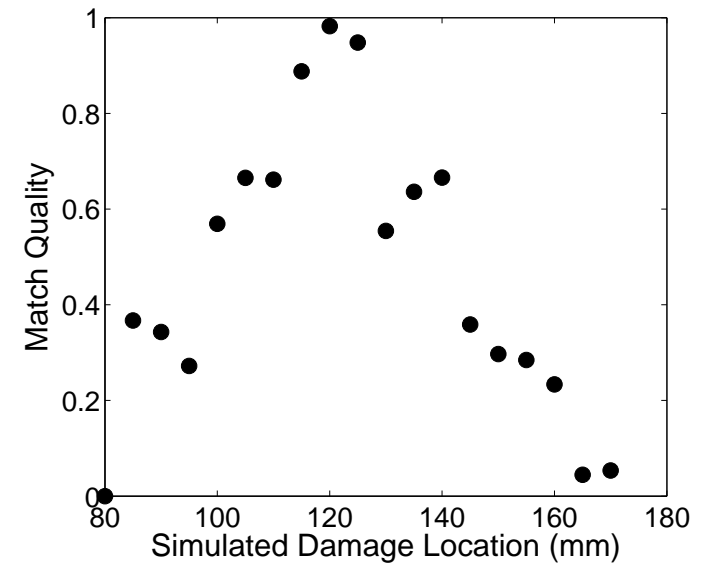

(a) Location identification - damage at $x=125 \mathrm{~mm}$

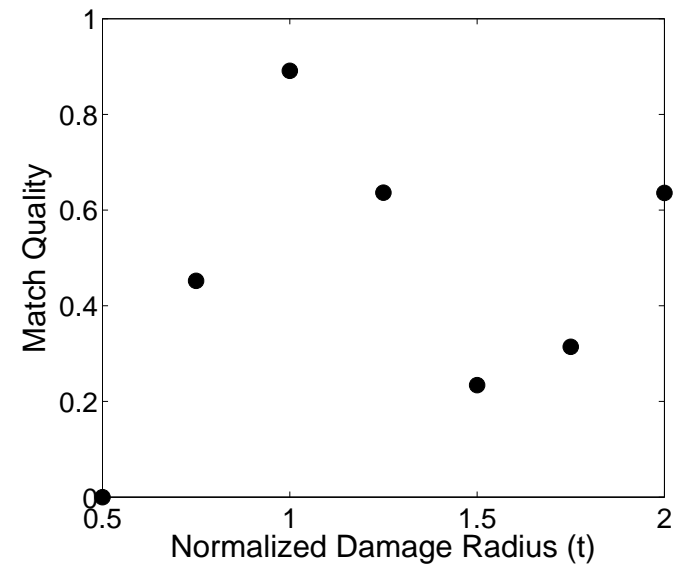

(b) Size identification - damage size $r=1 t$

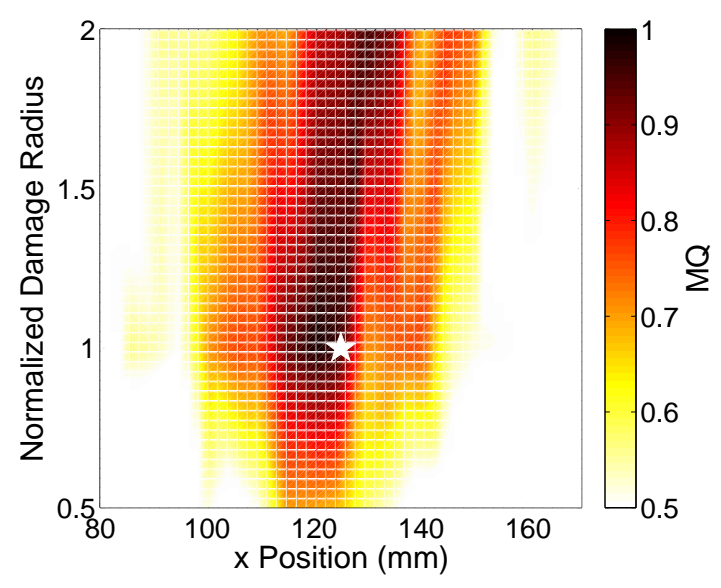

(c) Combined identification

Figure 15. Matching pursuit algorithm characterization of experimental hole damage in an aluminum plate. Actual damage size of $r=1 t$ located at $x=125 \mathrm{~mm}$ (1-D analysis, sensor 1 only).

\section{Matching Pursuit Algorithm: 2-D Scenario}

The next step in evaluating the matching pursuit algorithm involved extending the library of possible damage locations to a 2-D region. This analysis concentrated on $r=1 t$ through-thickness hole damage in the cross-ply laminate, and proper location of the hole damage was the primary focus. A schematic showing the location of the actuator, sensors, and possible damage locations is included in Fig. 17. For this scenario, two possible actuator locations were simulated. Five sensors were modeled around each actuator. For each possible damage site, simulations were conducted when each actuator was active. Based on the previous conclusion that sensors in the pitch-catch configuration were not optimally placed for this matching pursuit algorithm, only the sensors immediately surrounding the respective actuator were active during the simulations.

\section{A. 2-D Analysis Using a Single Sensor}

The first stage in the 2-D analysis of the algorithm was to evaluate its location capabilities when only data from sensor 1, resulting from A1 actuation, were used. The results for this scenario are shown in Fig. 18. In each of the sub-figures, the darker areas represent locations for which the algorithm predicted a closer match to the actual damage difference signal. The active sensor and actuator positions are also shown on the figures. With only the data from the single sensor, the algorithm produced mixed results. Figure 18 


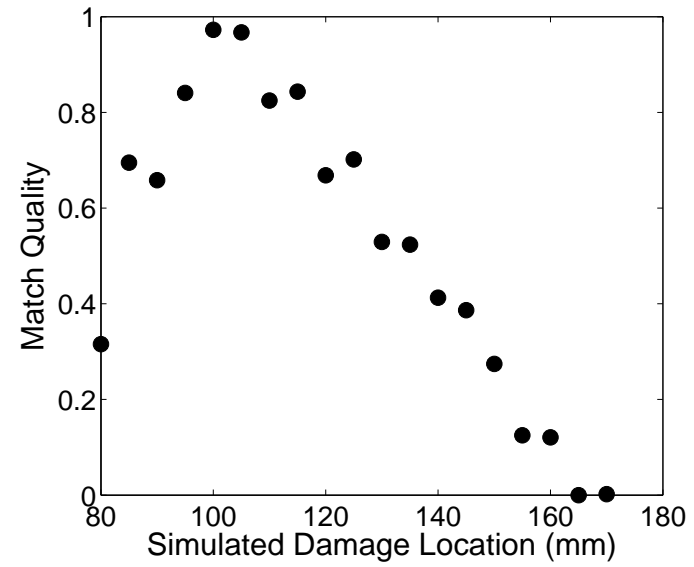

(a) Location identification - damage at $x=125 \mathrm{~mm}$

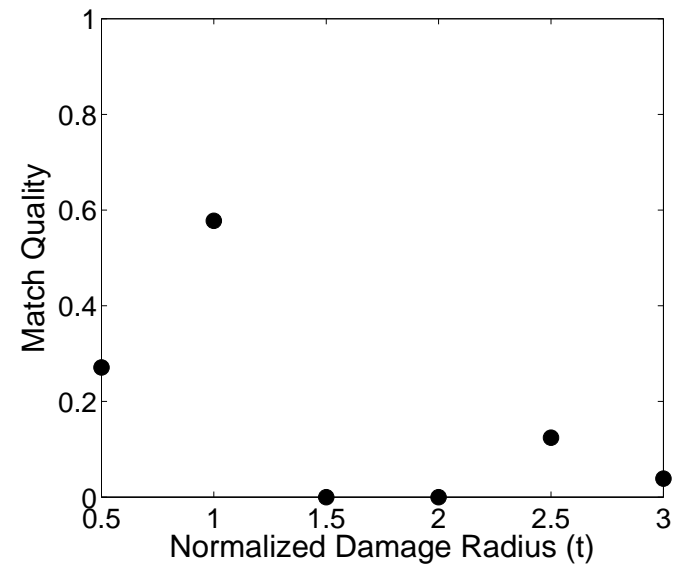

(b) Size identification - damage size $r=1 t$

Figure 16. Matching pursuit algorithm experimental damage characterization for a cross-ply laminate (1-D analysis, sensor 1 only).

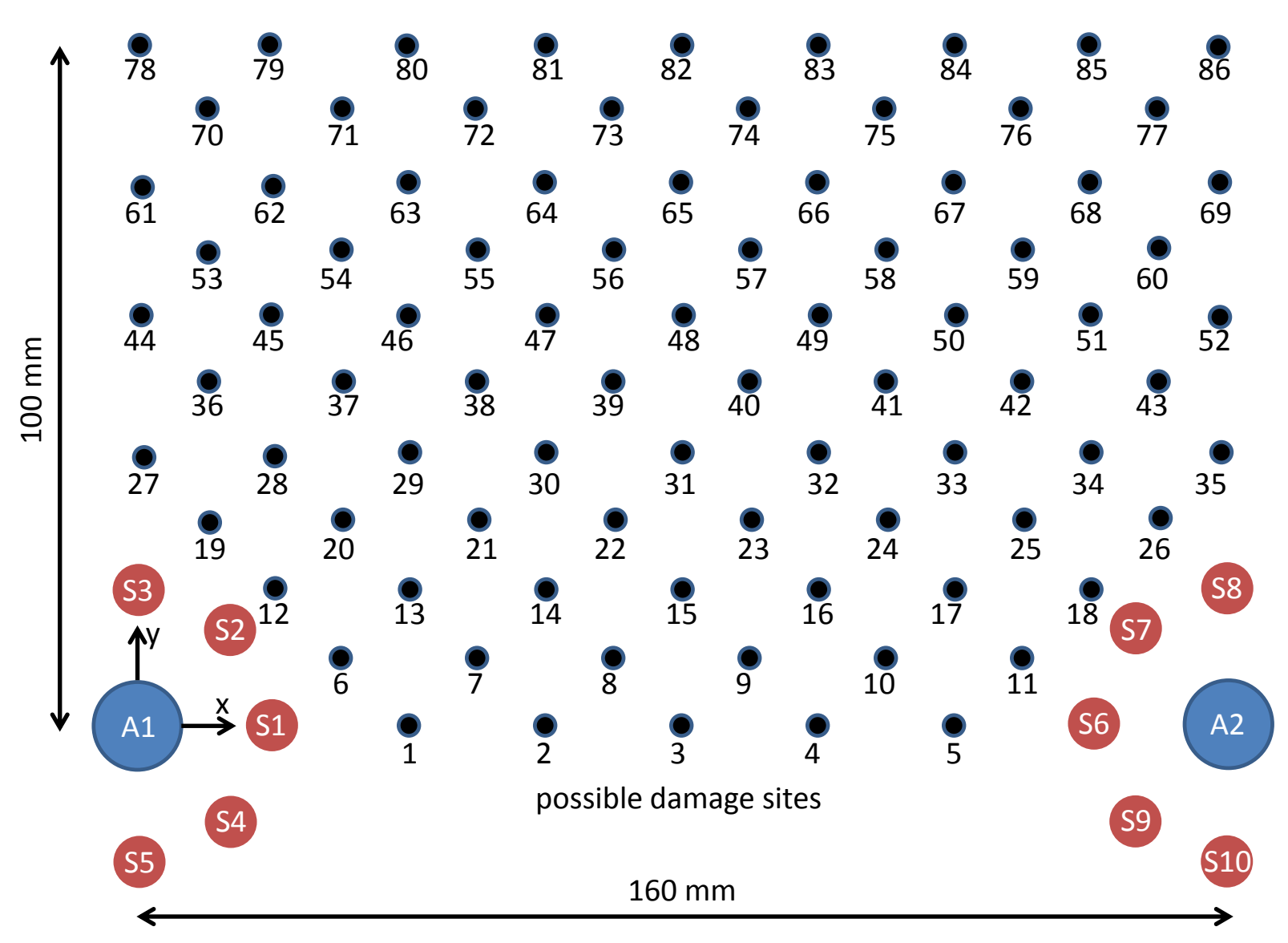

Figure 17. Schematic for 2-D matching pursuit analysis showing actuator positions (A1, A2), sensor configuration $(\mathrm{S} 1, \mathrm{~S} 2, \ldots, \mathrm{S} 10)$, and possible locations included in damage library. 


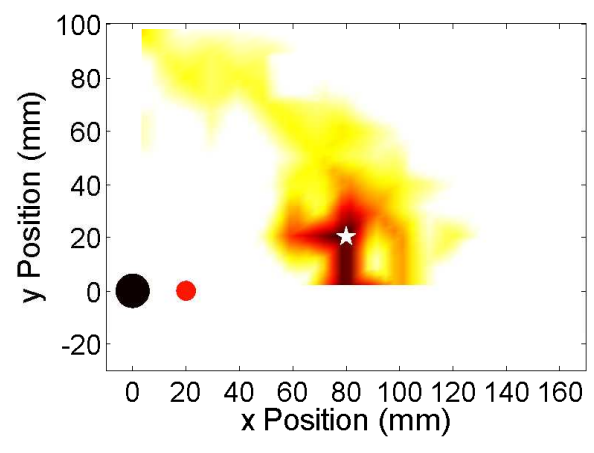

(a) Damage at $(80,20) \mathrm{mm}$

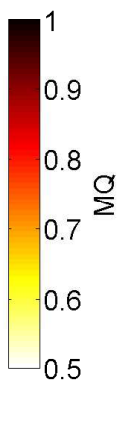

$\frac{0}{2}$

0.6

0.5

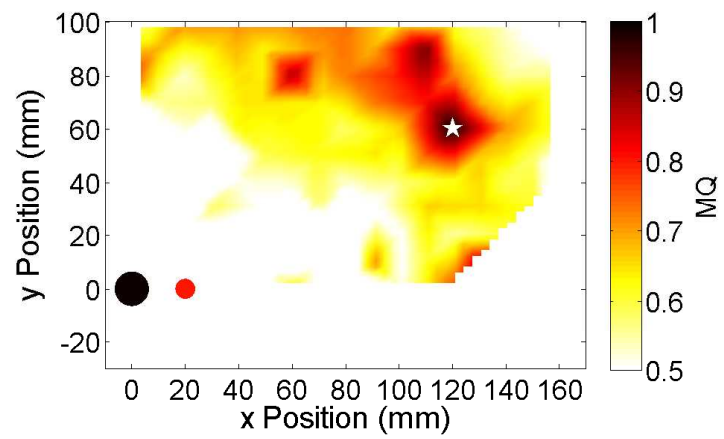

(b) Damage at $(120,60) \mathrm{mm}$

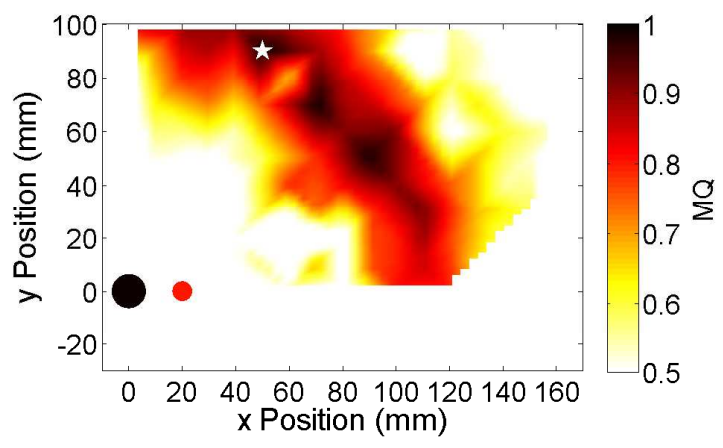

(c) Damage at $(50,90) \mathrm{mm}$

Figure 18. Matching pursuit algorithm damage location results for a cross-ply laminate using only sensor S1 (2-D scenario).

(a) shows one of the best cases, where the damage was located at the coordinates $(80,20)$ with respect to actuator A1, measured in millimeters. For this hole location, the algorithm produced a very accurate location even with only one sensor. A moderately successful result can be seen in Fig. 18 (b), which corresponds to a damage location of $(120,60) \mathrm{mm}$. Here, the algorithm correctly predicted the simulation corresponding to the damage location as the best match, but there were other locations that produced close matches as well. Finally, results for damage located at $(50,90) \mathrm{mm}$ are included in Fig. 18 (c). The algorithm indicated that numerous simulations were close matches to the actual damage signal, and the locations for the damage in these simulations formed a large area. In this case, using only one sensor did not provide satisfactory results.

A numerical comparison of these three models was obtained by calculating a predicted location for the damage based on the 4 simulations with the highest reported match quality. The predicted $x$ position was calculated using:

$$
x_{\text {predicted }}=\frac{\sum_{j=1}^{4}\left(x_{\text {sim }}(j)\right)(M Q(j))}{\sum_{j=1}^{4} M Q(j)}
$$

A similar equation was used to calculate the predicted $y$ position. The results for the three trials shown in Fig. 18 are included in Table 2.

\section{B. Effect of Using Multiple Sensor and Actuator Locations}

Because one sensor alone did not reliably locate the damage in all cases, additional sensors were added to the algorithm to aid in locating the damage. This involved running the matching pursuit algorithm individually 
Table 2. Matching pursuit algorithm damage location results for a cross-ply laminate using only sensor S1 (2-D scenario).

\begin{tabular}{cccc}
\hline Trial & Actual Location $(\mathrm{mm})$ & Predicted Location $(\mathrm{mm})$ & Error $(\mathrm{mm})$ \\
\hline 1 & $(80,20)$ & $(77.5,16.4)$ & 4.3 \\
2 & $(120,60)$ & $(105.3,60.7)$ & 14.7 \\
3 & $(50,90)$ & $(79.4,60.6)$ & 41.5 \\
\hline
\end{tabular}

for each sensor, and then averaging the results of each of the sensors included in the analysis.

The effect of including multiple sensors in the algorithm is illustrated in Fig. 19 for damage located at (50, 90) $\mathrm{mm}$. The previously shown case where only sensor S1 was used is shown again in sub-figure (a). In subfigure (b), results are shown for the algorithm when each of the five sensors (S1-S5) surrounding actuator A1 were used. It is evident in this figure that many of the incorrect damage locations have lightened in intensity, indicating their match quality has diminished. A different approach was to include both actuators with only one sensor associated with each, as shown in sub figure (c). In this case, actuator A1 was used with sensor S1, and actuator A2 was used with sensor S6. Even better results were obtained when both actuators and each of their corresponding sensors were used. In sub-figure (d), the results are for the case where sensors S1 through S5 were used for actuator A1 excitation and sensors S6 through S10 were used for actuator A2 excitation. When the results for all 10 sensors were averaged, an accurate location was reported without any regions of conflicting matches. Table 3 shows how the predicted hole location improved in accuracy

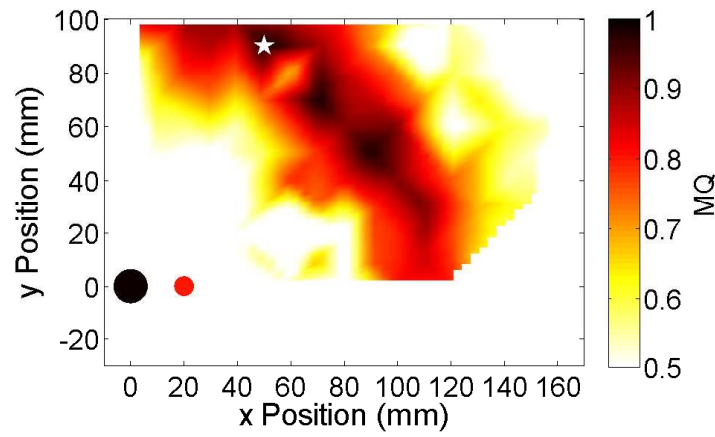

(a) Sensor 1 Only

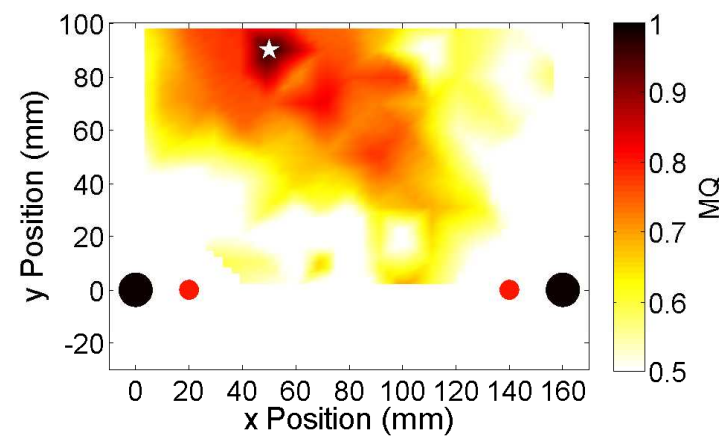

(c) Sensors 1 and 6

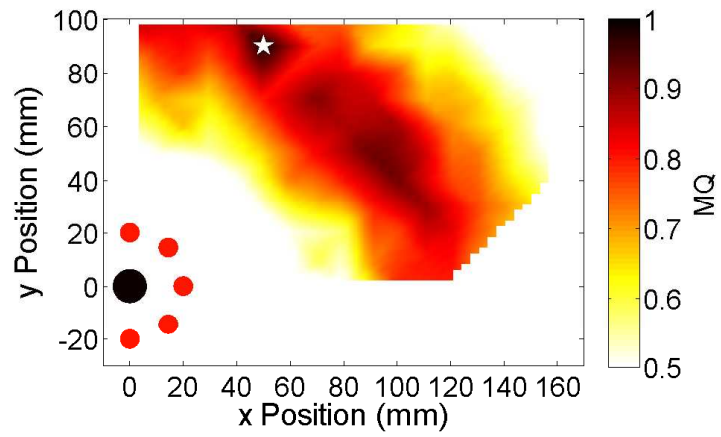

(b) Sensors 1 through 5

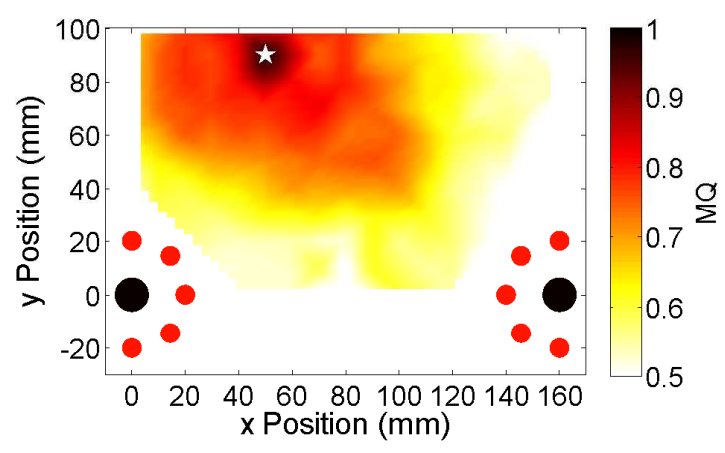

(d) Sensors 1 through 10

Figure 19. Improvement in matching pursuit algorithm location results for a cross-ply laminate when data from multiple sensors are used (2-D scenario with hole at $(50,90) \mathrm{mm})$. 
Table 3. Improvement in matching pursuit algorithm location results for a cross-ply laminate when data from multiple sensors are used (2-D scenario with hole at $(50,90) \mathrm{mm})$.

\begin{tabular}{ccc}
\hline Case & Predicted Location $(\mathrm{mm})$ & Error $(\mathrm{mm})$ \\
\hline A1/S1 & $(79.4,60.6$ & 41.5 \\
A1/S1-S5 & $(77.0,63.0)$ & 38.2 \\
A1/S1; A2/S6 & $(61.8,78.2)$ & 16.7 \\
A1/S1-S5; A2/S6-S10 & $(54.7,85.3)$ & 6.6 \\
\hline
\end{tabular}

as the number of actuators and sensors increased. It is notable that adding a second actuator/sensor pair had a much more significant effect on location accuracy than increasing the number of sensors used with a particular actuator from 1 to 5 .

Damage location results from the analysis of four additional damage sites are included in Fig. 20. All 10 sensors were used in each of these cases, and in each case the matching pursuit algorithm was able to locate the hole damage to within $4 \mathrm{~mm}$ when considering the four best-matching simulations. Numerical results are shown in Table 4.

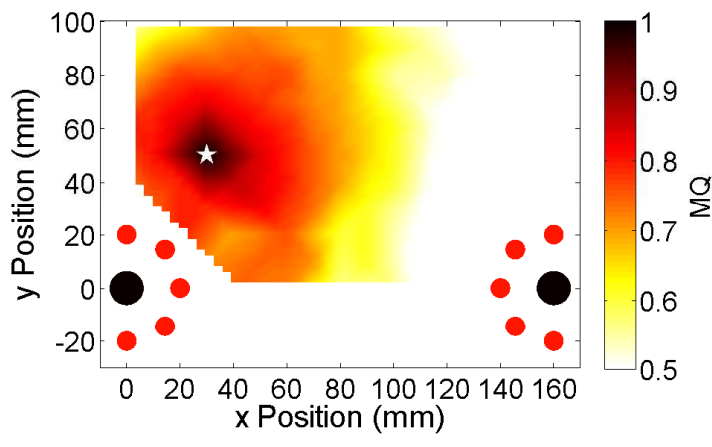

(a) Damage at site $(30,50) \mathrm{mm}$

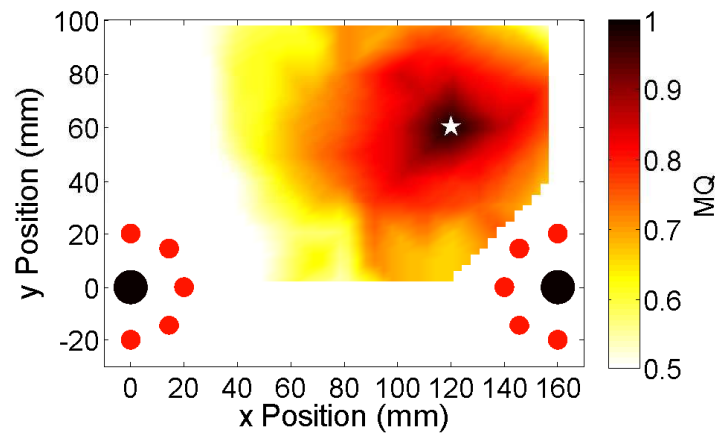

(c) Damage at site $(120,60) \mathrm{mm}$

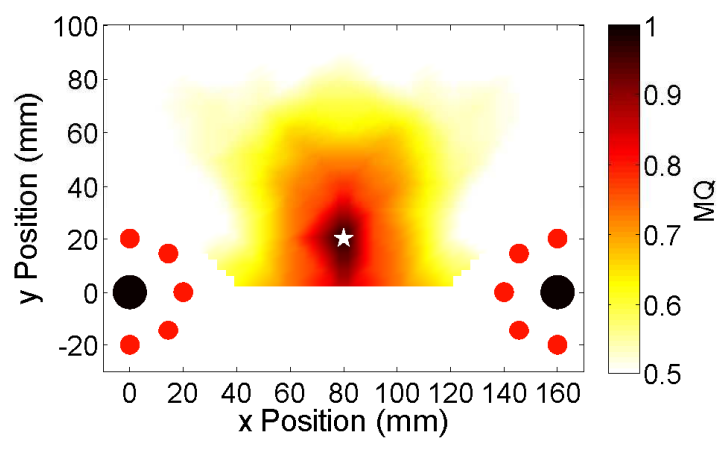

(b) Damage at site $(80,20) \mathrm{mm}$

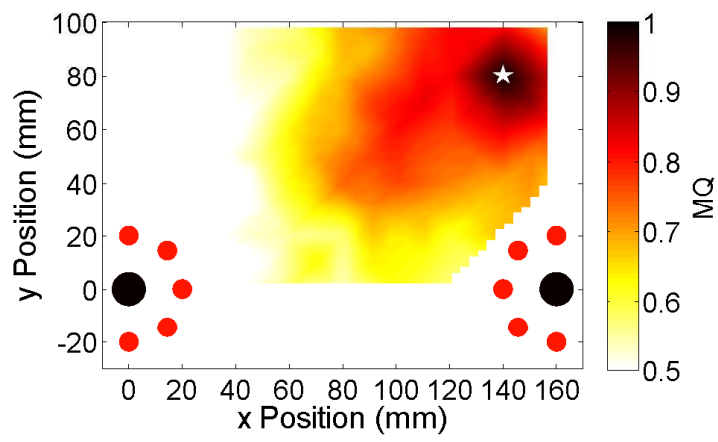

(d) Damage at site $(140,80) \mathrm{mm}$

Figure 20. Matching pursuit algorithm location results for a cross-ply laminate at various damage sites when all 10 sensors are used (2-D scenario). 
Table 4. Matching pursuit algorithm location results for a cross-ply laminate at various damage sites when all 10 sensors are used (2-D scenario).

\begin{tabular}{cccc}
\hline Trial & Actual Location $(\mathrm{mm})$ & Predicted Location $(\mathrm{mm})$ & Error $(\mathrm{mm})$ \\
\hline 1 & $(30,50)$ & $(32.4,52.3)$ & 3.4 \\
2 & $(80,20)$ & $(82.3,17.2)$ & 3.6 \\
3 & $(120,60)$ & $(119.9,59.9)$ & 0.2 \\
4 & $(140,80)$ & $(142.5,77.5)$ & 3.6 \\
\hline
\end{tabular}

\section{Location of Holes Not Included in Damage Library}

In each of the scenarios included to this point in the 2-D analysis, the actual damage location corresponded to a location captured by one of the damage library simulations. Because of this, each set of location results contained one location that was a perfect match. This section considers a few cases where the actual damage signal comes from a hole location not included in any of the library simulations. Ideally, the matching pursuit algorithm should identify the damage library simulations that are closest to these new damage points as the most likely matches for the damage. Figure 21 shows the location of the new damage points. LISA simulations were run to obtain the damage difference signals for each of the sensors for each of these damage locations. Each of these new difference signals were then used as the actual difference signals in the

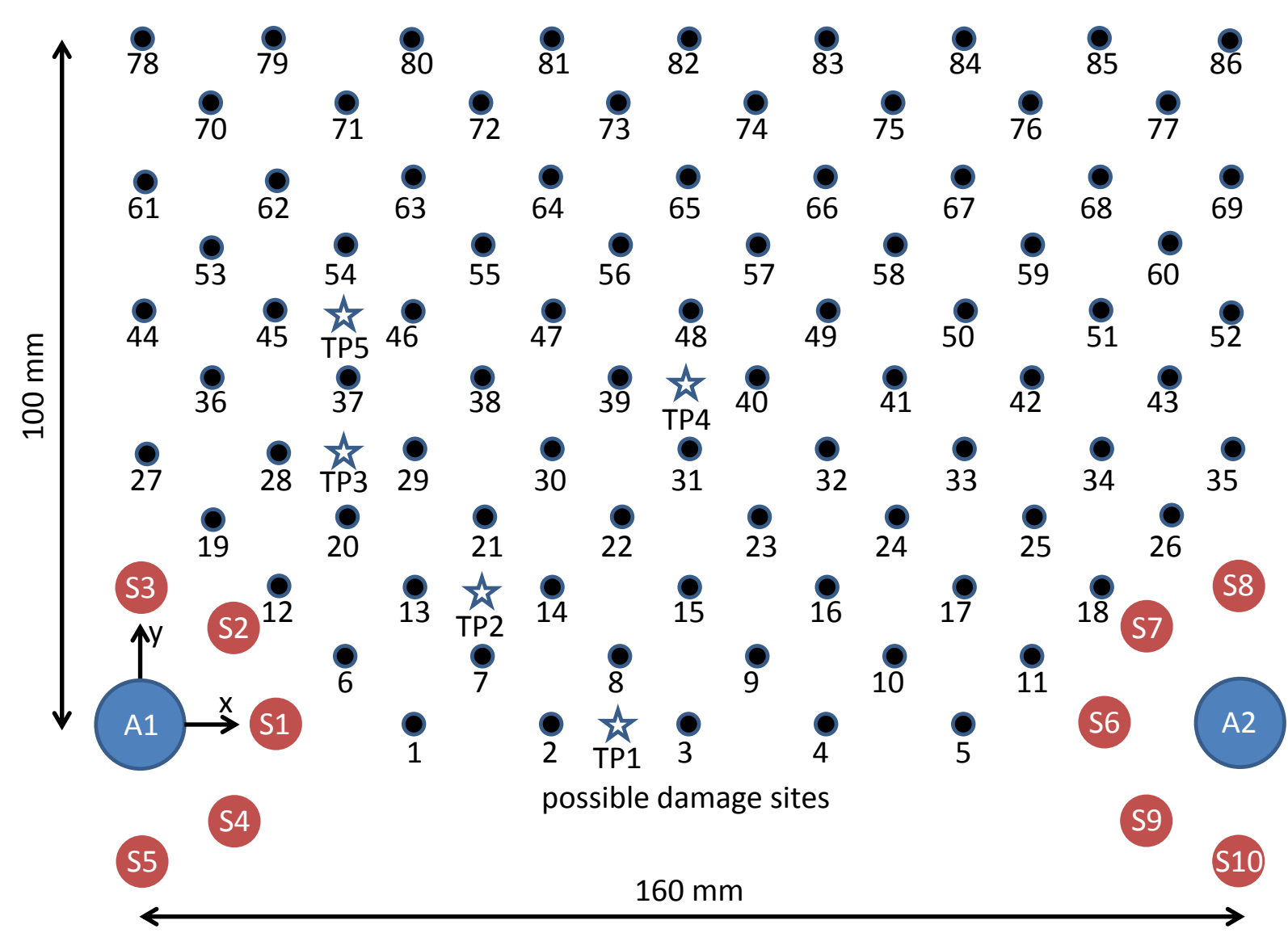

Figure 21. Location of non-collocated simulated test points (TP1-TP5) for 2-D analysis. 
Table 5. Matching pursuit algorithm location results for a cross-ply laminate with damage at non-simulated locations (2-D scenario).

\begin{tabular}{cccc}
\hline Trial & Actual Location $(\mathrm{mm})$ & Predicted Location $(\mathrm{mm})$ & Error $(\mathrm{mm})$ \\
\hline TP1 & $(70,0)$ & $(65.0,14.6)$ & 14.6 \\
TP2 & $(50,20)$ & $(57.4,22.5)$ & 7.6 \\
TP3 & $(30,40)$ & $(37.5,32.4)$ & 10.7 \\
TP4 & $(80,50)$ & $(80.0,49.9)$ & 0.1 \\
TP5 & $(30,60)$ & $(35.1,60.0)$ & 5.1 \\
\hline
\end{tabular}

algorithm. The graphical location results for these cases are shown in Fig. 22, and a numerical comparison is included in Table 5. For the cases considered, the location error ranged from $0.1 \mathrm{~mm}$ to $14.6 \mathrm{~mm}$.

\section{Summary}

This paper described a new damage characterization tool for guided wave SHM based on matching pursuits and LISA. Results from the 1-D scenario showed the algorithm's ability to predict the correct location of hole damage and the correct hole size in trial cases involving isotropic plates and cross-ply laminates. The algorithm was shown to be useful even in the case of excessive noise, and it was able to closely identify the location of experimental hole damage in an aluminum plate. It was determined that data from a sensor in the pulse-echo configuration were better suited to locating damage than data from a sensor in the pitch-catch configuration. It is expected that if both sensors are used, the location accuracy results will fall in the middle of the two individual sensor cases, since the data from the pitch-catch sensor will add uncertainty to the location prediction.

Demonstration of the algorithm in a 2-D scenario for a cross-ply laminate further reinforced its damage location capability. A sparse array of possible hole damage locations were included in the library of damage simulations. Results showed that using information from multiple sensors improved the location prediction, and including information from sensors associated with multiple actuators further improved the accuracy of the prediction. The algorithm was able to closely predict the location of simulated hole damage even when the exact hole location was not contained in the library of possible damage sites, with location errors ranging from $0.1 \mathrm{~mm}$ to $14.6 \mathrm{~mm}$ for the cases considered.

\section{Acknowledgments}

This work was sponsored by the National Rotorcraft Technology Center (NTRC) Vertical Lift/Rotorcraft Center of Excellence (VLRCOE) at the University of Michigan, with Mahendra J. Bhagwat as the technical monitor. Opinions, interpretations, conclusions, and recommendations are those of the authors and are not necessarily endorsed by the United States Government.

\section{References}

\footnotetext{
${ }^{1}$ Raghavan, A. and Cesnik, C. E. S., "Review of Guided Wave Structural Health Monitoring," The Shock and Vibration Digest, Vol. 39, No. 2, 2007, pp. 91-144.

${ }^{2}$ Staszewski, W. and Worden, K., "Signal Processing for Damage Detection," Health Monitoring of Aerospace Structures, 2004, pp. 163-206.

${ }^{3}$ Niethammer, M., Jacobs, L. J., Qu, J., and Jarzynski, J., "Time-frequency Representations of Lamb Waves," The Journal of the Acoustical Society of America, Vol. 109, No. 5, 2001, pp. 1841-1847.

${ }^{4} \mathrm{Kim}$, Y. Y. and Kim, E.-H., "Effectiveness of the Continuous Wavelet Transform in the Analysis of Some Dispersive Elastic Waves," The Journal of the Acoustical Society of America, Vol. 110, No. 1, 2001, pp. 86-94.

${ }^{5}$ Okabe, Y., Fujibayashi, K., Shimazaki, M., Soejima, H., and Ogisu, T., "Delamination Detection in Composite Laminates Using Dispersion Change Based on Mode Conversion of Lamb Waves," Smart Materials and Structures, Vol. 19, No. 11, 2010.

${ }^{6}$ Sohn, H., Park, G., Wait, J. R., Limback, N. P., and Farrar, C. R., "Wavelet-based Active Sensing for Delamination Detection in Composite Structures," Smart Materials and Structures, Vol. 13, No. 1, 2004, pp. 153-160.

${ }^{7}$ Mallat, S. G. and Zhang, Z., "Matching Pursuits with Time-frequency Dictionaries," Signal Processing, IEEE Transac-
} 


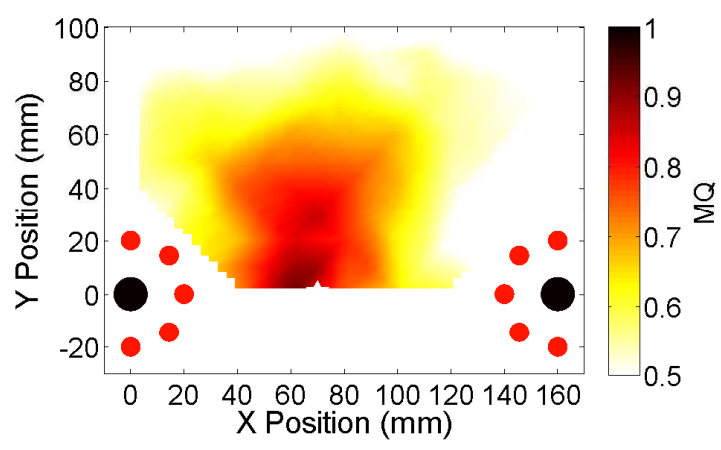

(a) Damage TP1 at site $(70,0) \mathrm{mm}$

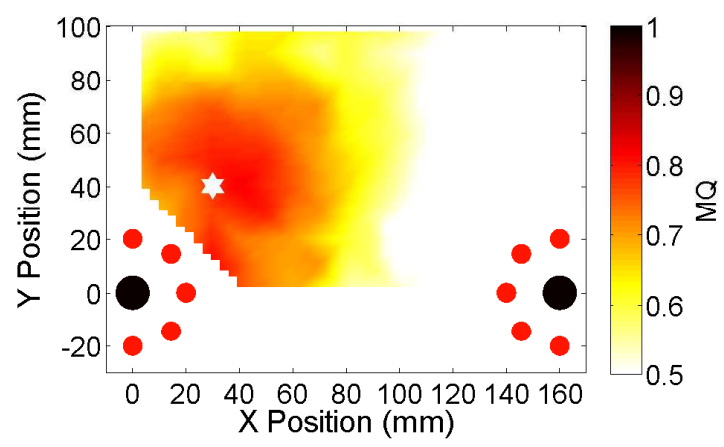

(c) Damage TP3 at site $(30,40) \mathrm{mm}$

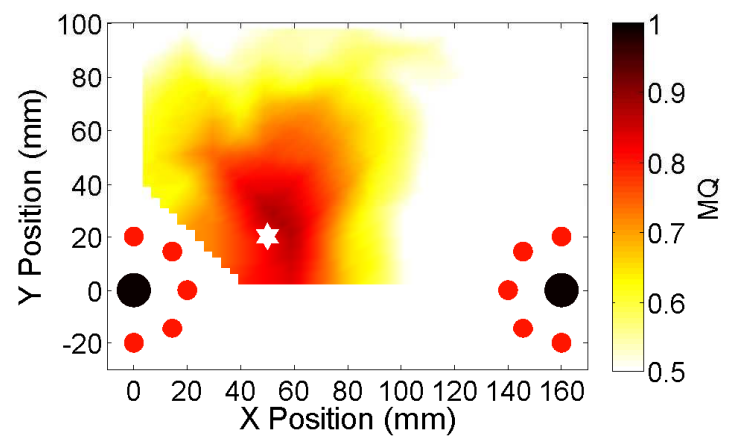

(b) Damage TP2 at site $(50,20) \mathrm{mm}$

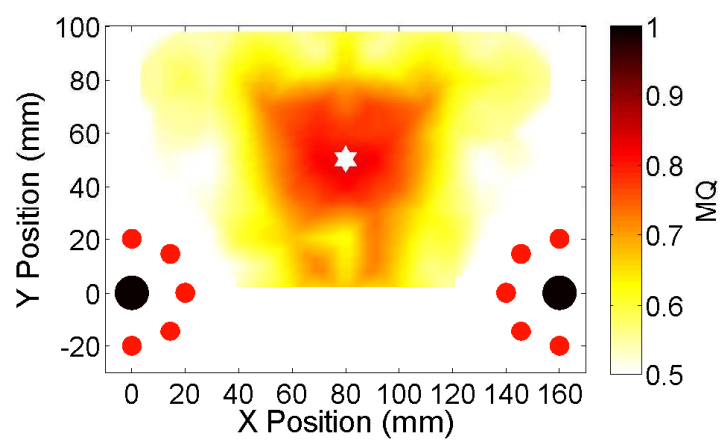

(d) Damage TP4 at site $(80,50) \mathrm{mm}$

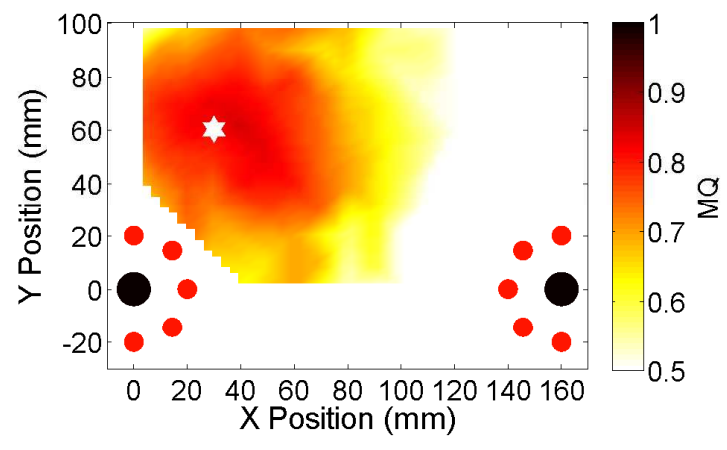

(e) Damage TP5 at site $(30,60) \mathrm{mm}$

Figure 22. Matching pursuit algorithm location results for a cross-ply laminate with damage at non-simulated locations (2-D scenario). 
tions on, Vol. 41, No. 12, 1993, pp. 3397-3415.

${ }^{8}$ Zhang, G., Zhang, S., and Wang, Y., "Application of Adaptive Time-frequency Decomposition in Ultrasonic NDE of Highly-scattering Materials," Ultrasonics, Vol. 38, No. 10, 2000, pp. 961-964.

${ }^{9}$ Hong, J.-C., Sun, K. H., and Kim, Y. Y., "The Matching Pursuit Approach Based on the Modulated Gaussian Pulse for Efficient Guided-wave Damage Inspection," Smart Materials and Structures, Vol. 14, No. 4, 2005, pp. 548-560.

${ }^{10}$ Das, S., Kyriakides, I., Chattopadhyay, A., and Papandreou-Suppappola, A., "Monte Carlo Matching Pursuit Decomposition Method for Damage Quantification in Composite Structures," Journal of Intelligent Material Systems and Structures, Vol. 20, No. 6, 2009, pp. 647-658.

${ }^{11}$ Vizzini II, A. J. and Chattopadhyay, A., "Lamb-wave-based Feature Extraction of Damage in a Stiffened Composite Panel Under Varying Temperature," Health Monitoring of Structural and Biological Systems, International Society for Optics and Photonics, 2012, pp. 83481P-83481P.

${ }^{12}$ Gribonval, R., "Fast Matching Pursuit with a Multiscale Dictionary of Gaussian Chirps," Signal Processing, IEEE Transactions on, Vol. 49, No. 5, 2001, pp. 994-1001.

${ }^{13}$ Raghavan, A. and Cesnik, C. E., "Guided-wave Signal Processing Using Chirplet Matching Pursuits and Mode Correlation for Structural Health Monitoring," Smart Materials and Structures, Vol. 16, No. 2, 2007, pp. 355-366.

${ }^{14}$ Agarwal, S. and Mitra, M., "Lamb Wave Based Damage Detection using Matching Pursuit and Support Vector Machine Classifier," SPIE Smart Structures and Materials+ Nondestructive Evaluation and Health Monitoring, International Society for Optics and Photonics, 2014, pp. 906424-906424.

${ }^{15}$ Delsanto, P. P., Whitcombe, T., Chaskelis, H. H., and Mignogna, R. B., "Connection Machine Simulation of Ultrasonic Wave Propagation in Materials I: the One-dimensional Case," Wave Motion, Vol. 16, 1992, pp. 65-80.

${ }^{16}$ Delsanto, P. P., Schechter, R. S., Chaskelis, H. H., Mignogna, R. B., and Kline, R., "Connection Machine Simulation of Ultrasonic Wave Propagation in Materials II: the Two-dimensional Case," Wave Motion, Vol. 20, 1994, pp. $295-314$.

${ }^{17}$ Delsanto, P. P., Schechter, R. S., and Mignogna, R. B., "Connection Machine Simulation of Ultrasonic Wave Propagation in Materials III: the Three-dimensional Case," Wave Motion, Vol. 26, 1997, pp. 329-339.

${ }^{18}$ Nadella, K. S. and Cesnik, C. E. S., "Numerical Simulation of Wave Propagation in Composite Plates," Health Monitoring of Structural and Biological Systems, Vol. 8348, International Society for Optics and Photonics, 2012.

${ }^{19}$ Sinor, M., "Numerical Modeling and Visualization of Elastic Waves Propagation in Arbitrary Complex Media," 8th Workship on Multimedia in Physics Teaching and Learning of the European Physical Society, Graz, 2004.

${ }^{20}$ Nadella, K. S. and Cesnik, C. E., "Local Interaction Simulation Approach for Modeling Wave Propagation in Composite Structures," CEAS Aeronautical Journal, 2013, pp. 1-14.

${ }^{21}$ Nadella, K. S. and Cesnik, C. E. S., "Piezoelectric Coupled LISA for Guided Wave Generation and Propagation," Health Monitoring of Structural and Biological Systems, Vol. 8695, International Society for Optics and Photonics, 2013.

${ }^{22}$ Obenchain, M. B. and Cesnik, C. E. S., "Hybrid Global Matrix/Local Interaction Simulation Approach for Wave Propagation in Composites," AIAA Journal, Vol. 52, 2014.

${ }^{23}$ Raghavan, A. and Cesnik, C. E. S., "Finite-dimensional Piezoelectric Transducer Modeling for Guided Wave Based Structural Health Monitoring," Smart Materials and Structures, Vol. 14, 2005, pp. $1448-1461$.

${ }^{24}$ Obenchain, M. B. and Cesnik, C. E. S., "Guided Wave Interaction with Hole Damage Using the Local Interaction Simulation Approach," Smart Materials and Structures, Vol. 23, No. 12, 2014

${ }^{25}$ Bacry, E., "LastWave 3.1 Software," http://www.cmap.polytechnique.fr/ bacry/LastWave, accessed 5 April 2014. 